\title{
- Density dependence on multiple spatial scales maintains spatial variation in both abundance and traits
}

\author{
Koen J. van Benthem ${ }^{\mathrm{a}, *}$, Meike J. Wittmann ${ }^{\mathrm{a}}$ \\ ${ }^{a}$ Department of Theoretical Biology, Bielefeld University, Bielefeld, Germany
}

\section{Abstract}

Population density affects fitness through various processes, such as mate finding and competition. The fitness of individuals in a population can in turn affect its density, making population density a key quantity linking ecological and evolutionary processes. Density effects are, however, rarely homogeneous. Different life-history processes can be affected by density over different spatial scales. In birds, for example, competition for food may depend on the number of birds nesting in the direct vicinity, while competition for nesting sites may occur over larger areas. Here we investigate how the effects of local density and of density in nearby patches can jointly affect the emergence of spatial variation in abundance as well as phenotypic diversification. We study a two-patch model that is described by coupled ordinary differential equations. The patches have no intrinsic differences: they both have the same fitness function that describes how an individual's fitness depends on density in its own patch as well as the density in the other patch. We use a phase-space analysis, combined with a mathematical stability analysis to study the long-term behaviour of the system. Our results reveal that the mutual effect that the patches have on each other can lead to the emergence and long-term maintenance of a low and a high density patch. We then add traits and mutations to the model and show that different selection pressures in the high and low density patch can lead to diversification between these patches. Via eco-evolutionary feedbacks, this diversification can in turn lead to changes in the long-term population densities: under some parameter settings, both patches reach the same equilibrium density when mutations are absent, but different equilibrium densities when mutations are allowed. We thus show how, even in the absence of differences between patches, interactions between them can lead to 
differences in long-term population density, and potentially to trait diversification.

- Keywords: eco-evolutionary dynamics, population density, diversity, Allee effect,

7 density-dependent selection

\section{1. Introduction}

9 Population density affects many aspects of an individual's life, such as resource competi-

10 tion (Nicholson, 1957), parasite prevalence (Patterson and Ruckstuhl, 2013) and various aspects of the mating system, such as mate finding or competition for mating partners

12 (Gascoigne et al., 2009; Kokko and Rankin, 2006). These processes can in turn affect

13 lifetime reproductive success. For an individual it is thus advantageous to be adapted to

14 the density it experiences. For example, at high density, investing in resource competition

15 may pay off, whereas such an investment is futile when density is low. At low density,

$\mathbf{1 6}$ it may instead pay off more to invest in mate finding (Berec et al., 2018; Gascoigne

17 et al., 2009). Such scenarios where the relative fitness of traits changes with density are referred to as density-dependent selection, a concept that has a long history (see MacArthur and Wilson, 1967). Although density-dependent selection is challenging to demonstrate (Travis et al., 2013), there are several clear examples. In a field population of great tits, fast exploratory behavior appears to be favored at low density and slow exploratory behavior at high density (Nicolaus et al., 2016). In experiments on Drosophila (Mueller, 1997; Mueller et al., 1991), populations were exposed to different densities, to which they adapted, most likely through evolution. Adaptation to density has also been demonstrated in moths (Plodia interpunctella), where males in an experiment adapted their reproductive strategy to the density experienced as larvae (Gage, 1995). Adaptation to density is also supported by observed patterns, such as the observed higher male aggressiveness in fig wasp species that tend to occur at smaller densities where killing another male yields the largest relative benefits (Reinhold, 2003). An individual's fitness can be affected by population density at more than one spatial scale, a phenomenon we call multi-scale density dependence. Multi-scale density depen-

\footnotetext{
* Corresponding author

Email address: koen.vanbenthem@uni-bielefeld.de (Koen J. van Benthem ) Preprint submitted to Elsevier
} 
32 dence should arise naturally if fitness is the result of multiple processes, e.g. occurring at

33 different points in the life cycle. For example, birds may compete for high-quality nesting

34 sites on a larger scale at the beginning of the breeding season, and then after settling

35 on a nesting site compete for food more locally within their neighborhood (Rodenhouse

з6 et al., 2003). The effect of density may even be inverted depending on the spatial scale

37 (Courchamp et al., 2008, box 2.7). For example, in arid vegetation, there is long-range

38 competition for water, but also short-range faciliation because existing vegetation helps

39 to retain water (Rietkerk, 2004). Similarly, mussels compete for food but may also bene-

40 fit from a high local density, probably because it protects from waves (Gascoigne et al.,

$412005)$. In dogwood trees, when a focal patch is exposed to cicadas, the per capita number

42 of attacks decreases with the tree density in that patch. However, whether cicadas decide

43 to attack that patch, also depends on whether larger, more preferable, patches of trees

${ }_{44}$ are nearby (Cook et al., 2001). Individuals may thus be exposed to density effects at

45 different scales simultaneously.

46 Here, we study how multi-scale density dependence affects spatial patterns of popula-

47 tion density and variation in traits under density-dependent selection. We explore the

48 possibility of obtaining a stable state with high-density patches that are being inhabited

49 mostly by individuals that have a high density niche and low-density patches inhabited

so mostly by individuals adapted to low density. It has formerly been shown that spatial

51 variation in density can emerge in homogeneous deterministic models, for example due

52 to Allee effects (Gyllenberg and Hemminki, 1999), or due to the interplay of long-range

53 competition either with small-scale facilitation (van de Koppel et al., 2005) or disper-

54 sal (Bolker and Pacala, 1997; Bolker, 2003; Sasaki, 1997). While these previous models

55 have focused on ecological dynamics, we also include evolution of a trait under density-

56 dependent selection.

57 The potential for adaptation to density is nontrivial because of the eco-evolutionary

58 feedback loop (sometimes also referred to as eco-genetic feedback, Kokko and López-

59 Sepulcre, 2007) that it is embedded in: while density may affect lifetime reproductive

60 success, simultaneously changes in lifetime reproductive success also affect population

${ }_{61}$ density. The study of adaptation to spatial variation in density thus requires taking into

62 account evolutionary and ecological processes simultaneously. So far, however, spatial 
63 models concerning these feedback loops have mainly focused on dispersal (Govaert et al.,

64 2019). In our study, instead, we focus on the direct effect that patches can have on each

65 other's fitness.

66 We evaluate the capacity of multi-scale density dependent fitness to generate and main-

67 tain long-term differences in abundances between patches. Specifically, the fitness of an

68 individual in our model is affected not only by the local density, but also by the density

69 in a nearby patch. Such effects may emerge for example when a nearby patch attracts

70 predators, that then spill over to the focal patch. Our model also includes the possibility

71 of positive density dependence, which may occur for example when the nearby patch is

72 attracting pollinators. By including traits into the model, we then study how subpopula-

73 tions can adapt to their local density. For a plant population, for example, the investment

74 into defenses against predators relative to the investment into attracting pollinators may

75 be subject to density-dependent selection. However, simultaneously, the trait affects the

76 density, thereby allowing for eco-evolutionary dynamics. We explore the conditions under

77 which such a model can lead to diversification. Here we focus on allopatric diversification,

78 that is, the evolution of different trait values in each patch.

\section{2. Methods \& Results}

so 2.1. Model overview

${ }_{81}$ We consider a population living in a habitat with two patches (Fig. 1). The patches may

82 differ in the population density and in the trait distribution of the inhabiting individuals,

sз but are otherwise identical. In particular, we assume for simplicity that they have the

84 same area such that we can use density and population size or abundance interchangeably,

85 but note that the results do not depend on this assumption. We first consider an ecological

86 model where all individuals have the same trait value and there is no migration. We

${ }_{87}$ assume multi-scale density dependence in the sense that fitness in a patch depends not

${ }_{88}$ only on population density the patch itself, but also on the density in the other patch.

89 Next, we consider an eco-evolutionary model where individuals differ in a trait under

so density-dependent selection and eco-evolutionary feedbacks between population density 


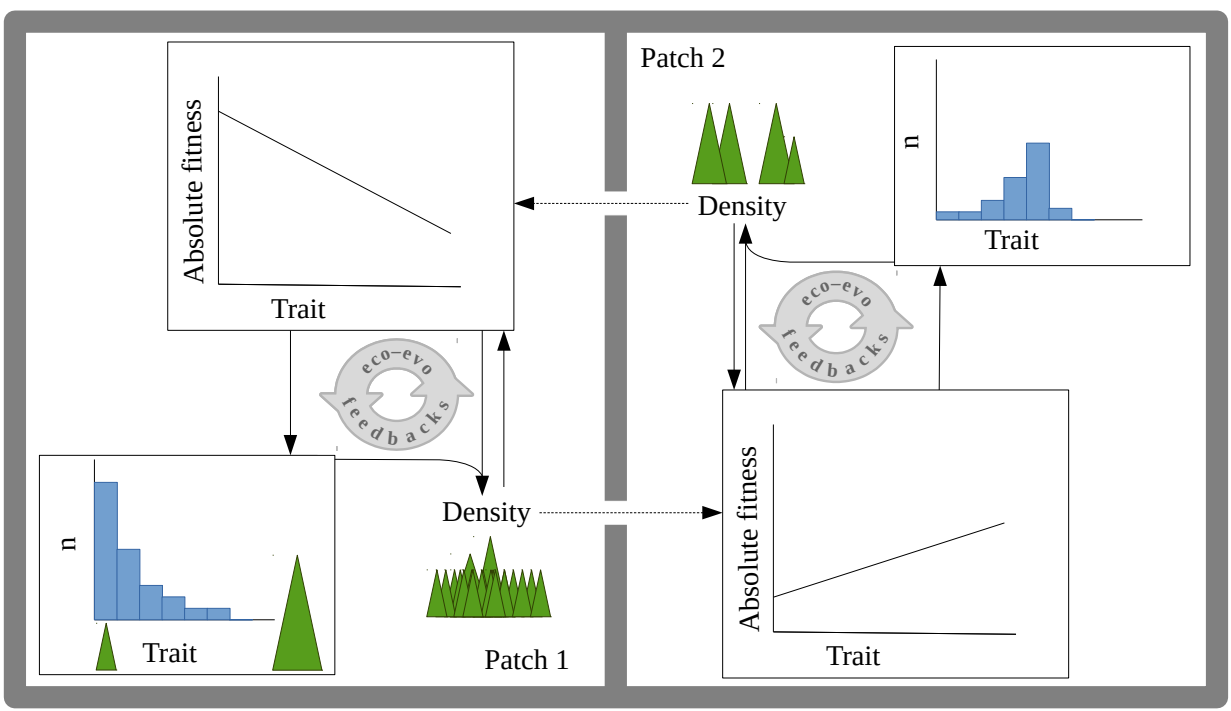

Figure 1: Overview of the ecological and evolutionary processes in our model. Absolute fitness differs between trait values and depends on density in the patch itself as well as on density in the other patch. Because of this density-dependent selection, the relationship between traits and fitness differs between patch 1 and 2. Absolute fitness then feeds back on population density but it also influences the evolution of the trait distribution (eco-evolutionary feedback).

91 and trait distribution emerge. Finally, we evaluate whether the outcomes are robust to

92 the inclusion of migration, stochasticity, and multi-locus genetics.

\section{2.2. Ecological model}

94 The population dynamics in the two patches are described by two coupled differential

95 equations:

$$
\begin{aligned}
& \frac{d N_{1}}{d t}=f\left(N_{1}, N_{2}\right) \cdot N_{1}, \\
& \frac{d N_{2}}{d t}=f\left(N_{2}, N_{1}\right) \cdot N_{2},
\end{aligned}
$$

96 with $N_{i}$ the density in patch $i$ and $f$ the per-capita growth rate or fitness function. Fitness

97 depends on the abundances in both patches and a set of coefficients $c_{\alpha}(\alpha \in[0,1,2,3,4])$ :

98

$$
f\left(N_{1}, N_{2}\right)=c_{0}+c_{1} N_{1}+c_{2} N_{2}+c_{3} N_{1}^{2}+c_{4} N_{2}^{2} .
$$


The coefficient $c_{0}$ represents the intrinsic growth rate in an empty habitat. The linear coefficients $c_{1}$ and $c_{2}$ characterize the response to increasing density in the own and other patch, respectively, while these densities are still low. The quadratic coefficients $c_{3}$ and $c_{4}$ for response to the own and the other patch become increasingly important as densities increase and thus determine the high-density behaviour of the system. Individuals in this model experience density effects at two spatial scales. Density of their respective own patch influences fitness via the second and fourth term, and density in the respective other patch influences fitness via the third and fifth term. Both patches behave equally and exchanging their labels would not affect the results. Note that our model is mathematically speaking a special case of the model developed by Gerla and Mooij (2014) for the interaction between competing species in a single patch. With this different interpretation, their results are in line with parts of our results for the ecological model, as discussed below in more detail.

By choosing the parameters $c_{\alpha}$, our model can represent various scenarios. Here, we focus mainly on negative values for $c_{3}$ and $c_{4}$ to prevent populations from growing to infinity. When only considering the effect of the 'own' density on fitness, e.g. in patch 1, and keeping the density of the other patch fixed, $\frac{\partial f_{1}}{\partial N_{1}}=0$ when $N_{1}=-\frac{c_{1}}{2 c_{3}}$. Since $\frac{\partial^{2} f_{1}}{\partial N_{1}^{2}}=2 c_{3}$, this point is a maximum if $c_{3}<0$. If $c_{1}$ is negative, the maximum is below zero and fitness decreases with $N_{1}$ everywhere. If $c_{1}$ is positive, the fitness maximum will be at a positive value of $N_{1}$. Below the abundance at the extremum, per-capita fitness increases with increasing abundance. Hence, the system exhibits an Allee effect (Courchamp et al., 2008). At high densities, above the density at the extremum, the relation is inverted and percapita fitness decreases with increasing abundance, representing for example increasing resource competition or aggression. Depending also on the other parameters, the Allee effect with positive $c_{1}$ might be strong with a negative per-capita growth rate at small densities or weak with a reduced but still positive per-capita growth rate at small densities (Courchamp et al., 2008). Similarly, when fixing the local density, fitness increases with the density in the other patch below $-\frac{c_{2}}{2 c_{4}}$ and decreases with density in the other patch above this value. Thus we can get either negative density dependence with respect to density in the other patch, or an analogue of a weak or strong Allee effect with respect to the density in the other patch. Of course all combinations of density-dependence sce- 


$$
\begin{aligned}
& c_{0}+c_{1} N_{1}+c_{2} N_{2}+c_{3} N_{1}^{2}+c_{4} N_{2}^{2}=0, \\
& c_{0}+c_{1} N_{2}+c_{2} N_{1}+c_{3} N_{2}^{2}+c_{4} N_{1}^{2}=0 .
\end{aligned}
$$

In the $\left(N_{1}, N_{2}\right)$ phase plane, each of these equations corresponds to a conical section. Under our standard assumption that $c_{3}$ and $c_{4}$ are negative such that populations cannot grow to infinity, the isoclines are ellipses. The ellipse corresponding to the isocline of patch are positive in the interior of the ellipse and negative outside the ellipse.

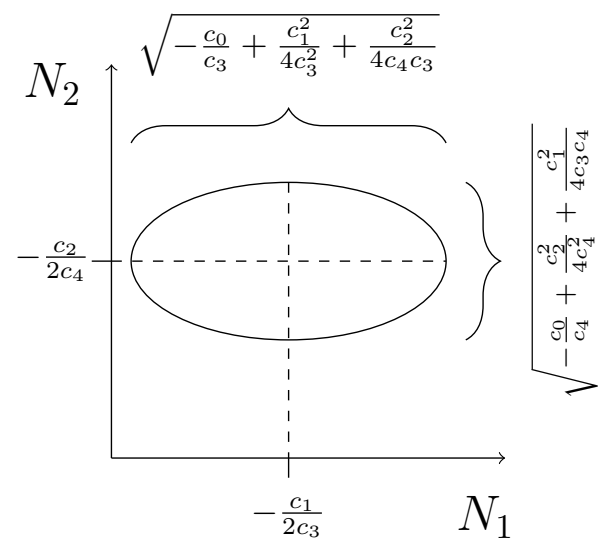

Figure 2: Geometric representation of the isocline. Isocline properties depend on ratios between coefficients only. Due to the isoclines containing no multiplication of $N_{1}$ and $N_{2}$, the axes of the ellipse are parallel to the $N_{1}$ - and $N_{2}$-axis. 
(a)

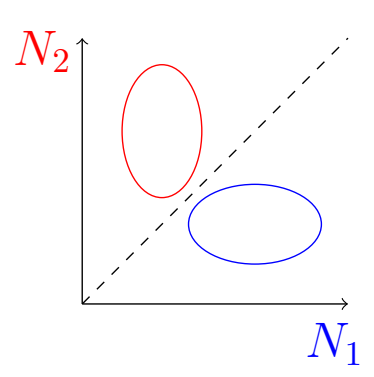

(b)

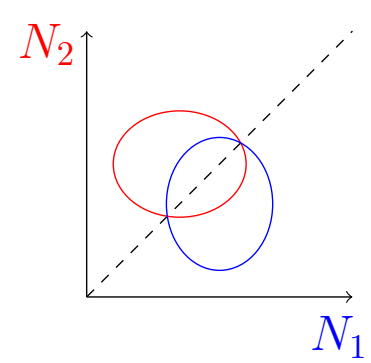

(c)

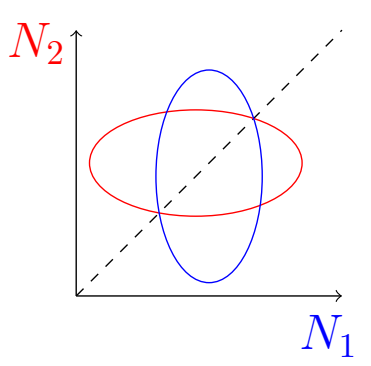

Figure 3: Possible number of intersections for two mirroring ellipses. This figure only illustrates the isoclines where $f_{i}=0$, the isoclines for $N_{i}=0$ correspond to the $\mathrm{x}$ and $\mathrm{y}$-axes and the ellipses can also intersect these.

The values of the equilibria can be obtained by solving equations 4 and 5 simultaneously. 
165 First, we subtract the two equations from each other and obtain:

$$
\left(c_{1}-c_{2}\right) N_{1}+\left(c_{2}-c_{1}\right) N_{2}+\left(c_{3}-c_{4}\right) N_{1}^{2}+\left(c_{4}-c_{3}\right) N_{2}^{2}=0
$$

166

which can be rewritten as:

$$
\left(c_{1}-c_{2}\right)\left(N_{1}-N_{2}\right)+\left(c_{3}-c_{4}\right)\left(N_{1}+N_{2}\right)\left(N_{1}-N_{2}\right)=0 .
$$

This condition is fulfilled when either of the two factors is zero, that is if $N_{1}=N_{2}$ or

$$
N_{1}+N_{2}=-\frac{c_{1}-c_{2}}{c_{3}-c_{4}}
$$

170 In the special case when $c_{3}=c_{4}$, the second equilibrium does not exist. Here, we are most

${ }_{171}$ interested in the second solution because it allows for equilibria where the two patches

172 contain a different density. We can now use this solution to eliminate $N_{2}$ from equations

1734 by setting $N_{2}=-N_{1}-\frac{c_{1}-c_{2}}{c_{3}-c_{4}}$ and regrouping:

$$
c_{0}-c_{2} \frac{c_{1}-c_{2}}{c_{3}-c_{4}}+c_{4}\left(\frac{c_{1}-c_{2}}{c_{3}-c_{4}}\right)^{2}+\left(c_{1}-c_{2}+2 c_{4} \frac{c_{1}-c_{2}}{c_{3}-c_{4}}\right) N_{1}+\left(c_{3}+c_{4}\right) N_{1}^{2}=0 .
$$

${ }_{174}$ We will solve this equation using the quadratic formula. Before we do so, we can slightly 175 simplify the higher order terms in the above equation by multiplying all terms with $\frac{c_{3}-c_{4}}{c_{3}+c_{4}}$ :

176

$$
c_{0} \frac{c_{3}-c_{4}}{c_{3}+c_{4}}-c_{2} \frac{c_{1}-c_{2}}{c_{3}+c_{4}}+c_{4} \frac{\left(c_{1}-c_{2}\right)^{2}}{\left(c_{3}-c_{4}\right)\left(c_{3}+c_{4}\right)}+\left(c_{1}-c_{2}\right) N_{1}+\left(c_{3}-c_{4}\right) N_{1}^{2}=0 .
$$

177 This can now be solved using the quadratic equation:

$$
N_{ \pm}=\frac{-\left(c_{1}-c_{2}\right) \pm \sqrt{\left(c_{1}-c_{2}\right)^{2}-4\left(c_{3}-c_{4}\right)\left(c_{0} \frac{c_{3}-c_{4}}{c_{3}+c_{4}}-c_{2} \frac{c_{1}-c_{2}}{c_{3}+c_{4}}+c_{4} \frac{\left(c_{1}-c_{2}\right)^{2}}{\left(c_{3}-c_{4}\right)\left(c_{3}+c_{4}\right)}\right)}}{2\left(c_{3}-c_{4}\right)}
$$

178 which can be written as:

$$
N_{ \pm}=\frac{-\left(c_{1}-c_{2}\right) \pm \sqrt{\left.c_{1}^{2}+c_{2}^{2}-2 c_{1} c_{2}-4 c_{0} \frac{\left(c_{3}-c_{4}\right)^{2}}{c_{3}+c_{4}}+4 c_{2} \frac{\left(c_{1}-c_{2}\right)\left(c_{3}-c_{4}\right)}{c_{3}+c_{4}}-4 c_{4} \frac{\left(c_{1}-c_{2}\right)^{2}}{\left(c_{3}+c_{4}\right)}\right)}}{2\left(c_{3}-c_{4}\right)} .
$$


179 Some rearrangement of these terms allows this expression to be written as:

$$
N_{ \pm}=\frac{-\left(c_{1}-c_{2}\right) \pm \sqrt{\frac{1}{\left(c_{3}+c_{4}\right)}\left(-4 c_{0}\left(c_{3}-c_{4}\right)^{2}+c_{1}^{2}\left(c_{3}-3 c_{4}\right)+2 c_{1} c_{2}\left(c_{3}+c_{4}\right)+c_{2}^{2}\left(c_{4}-3 c_{3}\right)\right)}}{2\left(c_{3}-c_{4}\right)} .
$$

194 The stability of the equilibria can be obtained through the Jacobian matrix

$$
\mathbf{J}=\left(\begin{array}{ll}
\frac{\partial f\left(N_{1}, N_{2}\right) N_{1}}{\partial N_{1}} & \frac{\partial f\left(N_{1}, N_{2}\right) N_{1}}{\partial N_{2}} \\
\frac{\partial f\left(N_{2}, N_{1}\right) N_{2}}{\partial N_{1}} & \frac{\partial f\left(N_{2}, N_{1}\right) N_{2}}{\partial N_{2}}
\end{array}\right)
$$

195 and hence

$$
\mathbf{J}=\left(\begin{array}{cc}
f\left(N_{1}, N_{2}\right)+N_{1} \frac{\partial f\left(N_{1}, N_{2}\right)}{\partial N_{1}} & N_{1} \frac{\partial f\left(N_{1}, N_{2}\right)}{\partial N_{2}} \\
N_{2} \frac{\partial f\left(N_{2}, N_{1}\right)}{\partial N_{1}} & f\left(N_{2}, N_{1}\right)+N_{2} \frac{\partial f\left(N_{2}, N_{1}\right)}{\partial N_{2}}
\end{array}\right) .
$$

196 At the nontrivial equilibrium, $f\left(N_{1}, N_{2}\right)=f\left(N_{2}, N_{1}\right)=0$ and thus:

$$
\mathbf{J}=\left(\begin{array}{ll}
N_{1} \frac{\partial f\left(N_{1}, N_{2}\right)}{\partial N_{1}} & N_{1} \frac{\partial f\left(N_{1}, N_{2}\right)}{\partial N_{2}} \\
N_{2} \frac{\partial f\left(N_{2}, N_{1}\right)}{\partial N_{1}} & N_{2} \frac{\partial f\left(N_{2}, N_{1}\right)}{\partial N_{2}}
\end{array}\right) .
$$




$$
N_{1}\left(c_{1}+2 c_{3} N_{1}\right)+N_{2}\left(c_{1}+2 c_{3} N_{2}\right)<0 .
$$

If both eigenvalues of this matrix, evaluated at an equilibrium of interest, have a negative characteristic equation, we find the two eigenvalues:

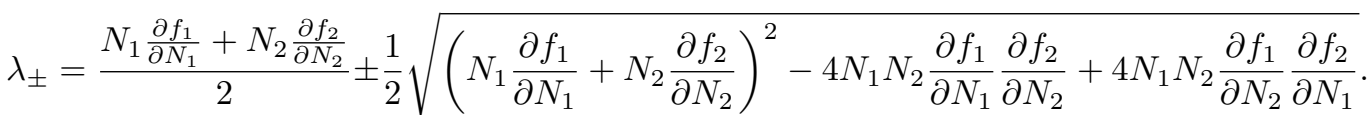

Here, we used the short-hand notation $f_{1}=f\left(N_{1}, N_{2}\right)$ and $f_{2}=f\left(N_{2}, N_{1}\right)$. After rearranging, we finally obtain

$$
\lambda_{ \pm}=\frac{N_{1} \frac{\partial f_{1}}{\partial N_{1}}+N_{2} \frac{\partial f_{2}}{\partial N_{2}}}{2} \pm \frac{1}{2} \sqrt{\left(N_{1} \frac{\partial f_{1}}{\partial N_{1}}-N_{2} \frac{\partial f_{2}}{\partial N_{2}}\right)^{2}+4 N_{1} N_{2} \frac{\partial f_{1}}{\partial N_{2}} \frac{\partial f_{2}}{\partial N_{1}}}
$$

To assess stability, it suffices to check the eigenvalue with the largest real part. Since the real part of $\lambda_{+}$is greater or equal to the real part of $\lambda_{-}$, the condition for stability thus becomes:

$$
\Re\left(\lambda_{+}\right)<0
$$

necessary but not sufficient condition for this is:

$$
N_{1} \frac{\partial f_{1}}{\partial N_{1}}+N_{2} \frac{\partial f_{2}}{\partial N_{2}}<0
$$

We use equation 20 to evaluate the stability of the equilibria from equation (14) by setting $N_{1}=N_{+}$and $N_{2}=N_{-}$(or vice versa). As noted above, these values remain the same as long as all coefficients keep their relative values and sign. Furthermore, if the fitness function is multiplied by a positive, real constant, the eigenvalues of the Jacobian matrix 1 also scale with this constant and hence the stability does not change (multiplication by 2 a positive number does not affect the sign). Hence a temporally varying environmental factor that acts by scaling the coefficients and hence the fitness function uniformly in both 4 patches, would not alter the equilibria nor their stability. Such environmental variation may however change the basin of attraction of equilibria. 


\subsubsection{Ecological model results}

Fig. 4 (top panels) shows an example system where at equilibrium both patches can have different densities. The left panel shows abundance time series, while the corresponding phase space trajectories are shown on the right. The colors refer to initial conditions (same color means same initial conditions). From the right panel it becomes clear that all trajectories end on an intersection between two isoclines. However, the specific equilibrium that the system reaches depends on its initial values for $N_{1}$ and $N_{2}$. The coefficients in the bottom panels were equal to those in the top panels, but with the effects of 'own' and 'other' patch exchanged $\left(c_{1} \leftrightarrow c_{2}\right.$ and $\left.c_{3} \leftrightarrow c_{4}\right)$. In this system, the equilibria where the patches contain different nonzero abundances are unstable. Instead, one of the equilibria with equal abundance in both patches is stable. Furthermore, additional equilibria, where one of the two patches goes extinct, have become stable.

The values of the coefficients determine which equilibria exist and which of these are stable. With equations 14, 19 and 20, it is possible to calculate the equilibria for any set of coefficients and evaluate their stability. The full parameter space is five-dimensional, but we evaluated the equilibria and their stability only at two-dimensional cross sections of that space (Fig. 5). Each cross section describes the effect of two of the coefficients, whilst keeping the remaining three coefficients at their value from the top panels of Fig. 4. The figure shows that most parameter combinations do not lead to an equilibrium in which both patches contain a different number of individuals. However, the region within which both patches may settle to different abundances is non-negligible. Small changes in the coefficients around the values from Fig. 4 are therefore not expected to lead to qualitative differences in the outcomes.

Above, we remarked that a necessary condition for the existence of meaningful asymmetric equilibria is that the strength of the response to the other patch is stronger than the response to the own patch in both linear and quadratic term, or weaker in both linear

and quadratic term. Here we have explored the parameter space around a point where the other patch has a stronger effect and thus we observe stable variation in abundance when $c_{2}>c 1$ (see 3rd row, 2nd column in Fig. 5) and when $\left|c_{4}\right|>\left|c_{3}\right|$ (4th row, 4th column in Fig. 5). Exploration of the $c_{1}-c_{0}$ parameter space $\left(4^{\text {th }}\right.$ row, $1^{\text {st }}$ column in Fig. 
5) reveals that stable spatial density variation should be possible also for cases where increasing density in the own patch has a negative effect even at low density $\left(c_{1}<0\right)$. Time series and isoclines for a parameter combination in this region are shown in Fig. 6. In this example, both $c_{1}$ and $c_{3}$ are negative, meaning that there is no Allee effect acting directly within the focal patch, although $c_{2}$ is still positive, leading to positive effects of density in the other patch on the fitness in the focal patch at low density in the other patch. Furthermore, all trajectories in this example converge to the asymmetric equilibria, which turn out to be the only stable equilibria.

\subsection{Eco-evolutionary model}

Long-term differences in population density may lead to diversification in traits under density-dependent selection, which may in turn affect the densities. In order to allow for such eco-evolutionary feedbacks, we now include a trait, $z$, that takes values between 0 and 1 . The trait affects the fitness through two additional terms: the first quantifies a density-independent effect of the trait value on the fitness $\left(c_{5} z\right)$, while the second describes a density-dependent effect of the trait value $\left(c_{6} z N_{i}\right)$ :

$$
f\left(z, N_{1}, N_{2}\right)=c_{0}+c_{1} N_{1}+c_{2} N_{2}+c_{3} N_{1}^{2}+c_{4} N_{2}^{2}+c_{5} z+c_{6} z N_{1} .
$$

Examples of traits whose fitness consequences are affected by density are investment in attributes for fighting or pheromone production for mate finding. Note that in this model, the selection on $z$ changes with the density in the own patch, but not with the density in the other patch.

Now, not only the population size, but also the trait distribution matters. We track the trait distribution by dividing the trait space into 100 discrete bins, with $z_{b}$ the trait value of bin $b$ and all the $z_{b}$ evenly spaced between 0 and 1 . The total abundance in patch 1 is simply the sum of the number of individuals in all size classes $b$ in the patch:

$$
N_{1}=\sum_{b=1}^{100} n_{1, b}
$$

The abundances $n_{1, b}$ change through reproduction, as described by the fitness function, as well as through mutations. We treat mutations deterministically, such that individuals 
(a)

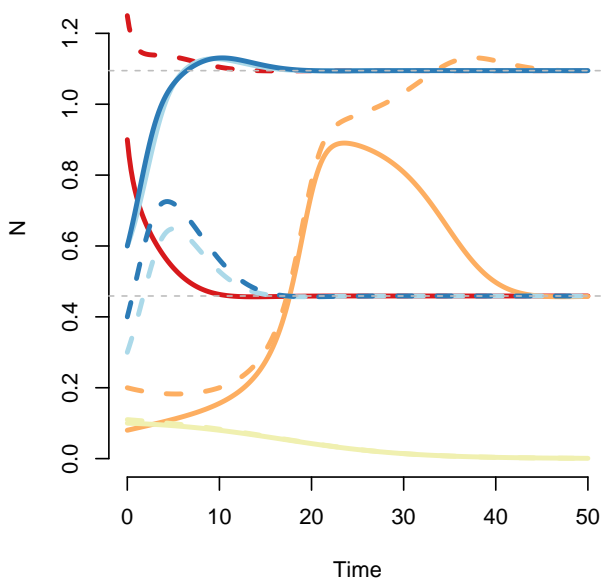

(c)

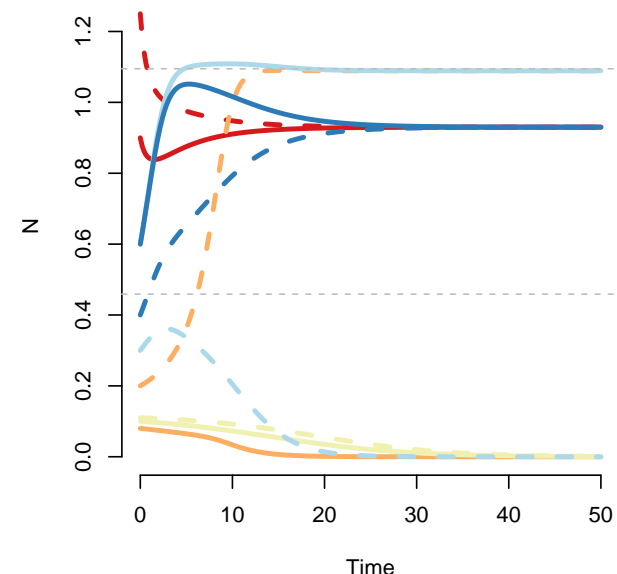

(b)

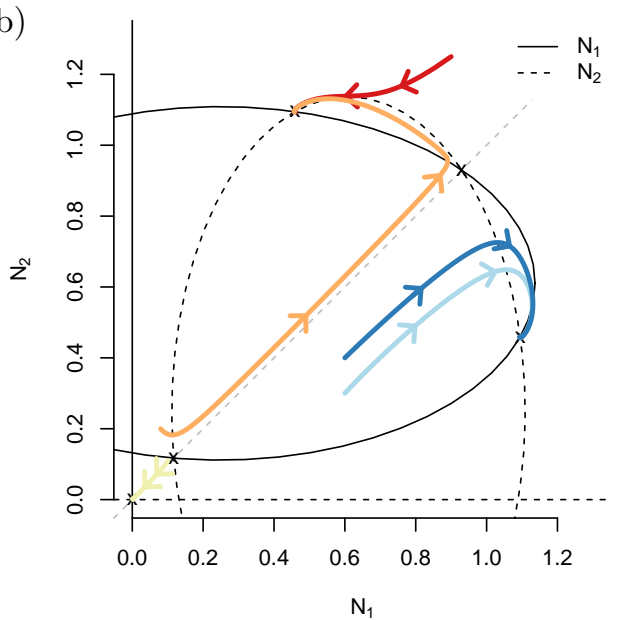

(d)

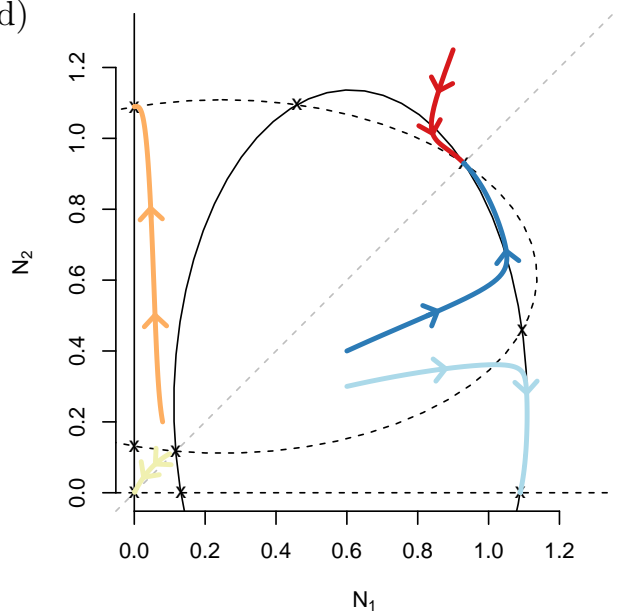

Figure 4: Example time series (a,c) and corresponding trajectories and isoclines in phase space (c,d). Different colors correspond to different initial values. The parameter values used for $(\mathrm{a}, \mathrm{b})$ were: $c_{0}=$ $-0.148, c_{1}=0.162, c_{2}=1.262, c_{3}=-0.326$, and $c_{4}=-1.034$. The parameters for $(\mathrm{c}, \mathrm{d})$ were $c_{0}=$ $-0.148, c_{1}=1.262, c_{2}=0.162, c_{3}=-1.034$, and $c_{4}=-0.326$. The bottom panels thus describe a system in which the effects of the 'own' patch and the 'other' patch have been exchanged. The ellipses also exchange identity, although their shape and intersection remain the same. The stability of the equilibria did change however. 
bioRxiv preprint doi: https://doi.org/10.1101/759415; this version posted September 8, 2019. The copyright holder for this preprint (which was not certified by peer review) is the author/funder, who has granted bioRxiv a license to display the preprint in perpetuity. It is made available under aCC-BY-ND 4.0 International license.

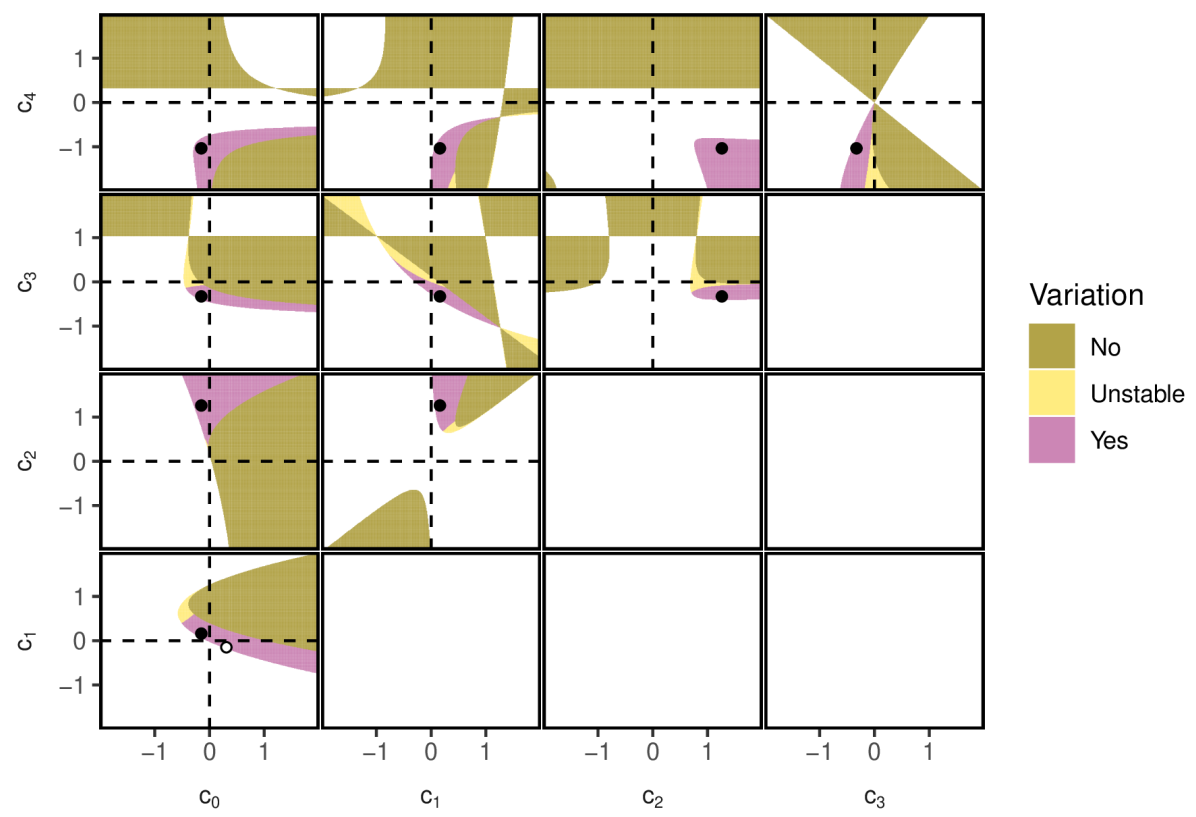

Figure 5: Regions in parameter space where density variation in space can be stable. Only the upper diagonal graphs are shown. Here, in the white regions, equation 14 returned equilibria with a nonzero imaginary part. In the 'No' region, the obtained equilibria were real, but either unreachable (at least one of them was negative) or the equilibria for $N_{1}$ and $N_{2}$ were the same. Finally, there were cases where the two equilibria were real, positive, and different. These cases were again subdivided in cases where the equilibrium was stable ('Yes') and where it was not ('Unstable'). The black dots correspond to the parameter settings that were used in the top panels of Fig. 4 . The white dot in the $c_{0}-c_{1}$ panel corresponds to the parameter setting of $c_{0}$ and $c_{1}$ in Fig. 6. 
(a)

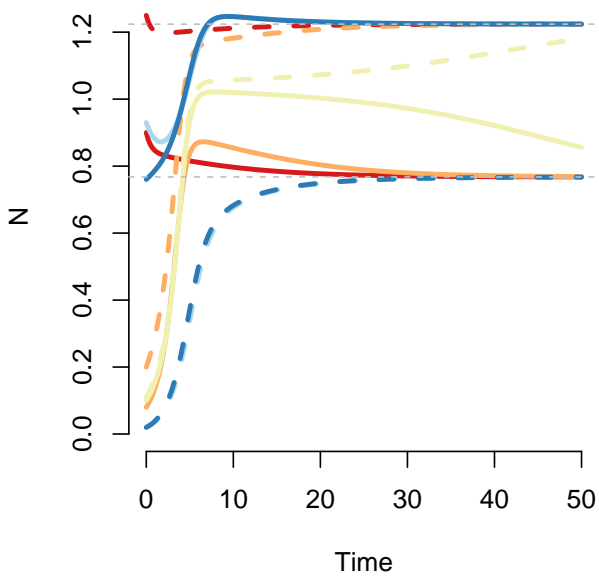

(b)

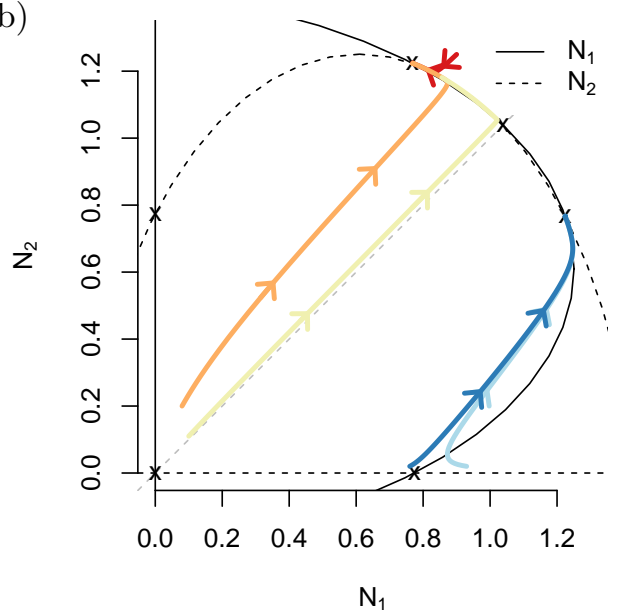

Figure 6: Example time series (a) and corresponding trajectories and isoclines in phase space (b). Different colors correspond to different initial values. The parameter values were: $c_{0}=0.31, c_{1}=-0.148$, $c_{2}=1.262, c_{3}=-0.326$, and $c_{4}=-1.034$.

${ }_{271}$ in bin $b$ mutates away to either bin $b-1$ or $b+1$ at a rate $\mu$. In the first and the last

272 bin, the trait value can only move in one direction, and accordingly, the mutation rate in 273 this bin is halved $\left(\frac{\mu}{2}\right)$. The dynamics of this system are described by a set of differential 274 equations:

$$
w_{1, b}=\left\{\begin{array}{ll}
\frac{d n_{1, b}}{d t}=f\left(z_{b}, N_{1}, N_{2}\right) n_{1, b}-\mu n_{1, b}+\frac{\mu}{2} n_{1, b-1}+\frac{\mu}{2} n_{1, b+1} & \text { if } 1<b<100 \\
\frac{d n_{1,1}}{d t}=f\left(z_{1}, N_{1}, N_{2}\right) n_{1,1}-\frac{\mu}{2} n_{1,1}+\frac{\mu}{2} n_{1,2} & \text { if } b=1 \\
\frac{d n_{1,100}}{d t}=f\left(z_{100}, N_{1}, N_{2}\right) n_{1,100}-\frac{\mu}{2} n_{1,100}+\frac{\mu}{2} n_{1,99} & \text { if } b=100
\end{array} .\right.
$$

275 The equations for the second patch are analogous. This yields 200 coupled differential 276 equations, that we initially solved numerically using the package deSolve in $\mathrm{R}$ ( $\mathrm{R}$ Core 277 Team, 2018; Soetaert et al., 2010).

\section{2.3.1. Equilibria} The numerically obtained equilibria were then compared to a direct calculation of the 280 equilibria when $\mu \rightarrow 0$. For a given combination of $N_{1}, c_{5}$, and $c_{6}$, the fitness function 281 is monotonic in $z$. If $c_{5}+c_{6} N_{1}>0$, larger trait values will be selected for (smaller trait 
282 values if $\left.c_{5}+c_{6} N_{1}<0\right)$. We therefore hypothesize the average trait value of a patch

$\mathbf{2 8 3}$ to end up at either boundary (0 or 1$)$. Since population density and thus the direction

${ }^{284}$ of selection can differ between patches, the two-patch system can potentially have four

$\mathbf{2 8 5}$ equilibria. We solved for the equilibria by solving the following four sets of two equations:

$$
\begin{aligned}
& f\left(0, N_{1}, N_{2}\right)=0 \text { and } f\left(0, N_{2}, N_{1}\right)=0, \\
& f\left(1, N_{1}, N_{2}\right)=0 \text { and } f\left(0, N_{2}, N_{1}\right)=0, \\
& f\left(0, N_{1}, N_{2}\right)=0 \text { and } f\left(1, N_{2}, N_{1}\right)=0, \\
& f\left(1, N_{1}, N_{2}\right)=0 \text { and } f\left(1, N_{2}, N_{1}\right)=0 .
\end{aligned}
$$

286 This procedure is equivalent to intersecting the two ellipses with $z=0$ and $z=1$ for the

${ }_{287}$ first patch with the two analogous ellipses of the other patch in all $2 \times 2$ combinations.

${ }_{288}$ The exception occurs when $c_{5}+c_{6} N_{i}=0$; in this case there is no selection on the trait 289 value and hence, the equilibrium has become independent of the trait value, and we

290 should still find it when $z=0$ or $z=1$. We used Mathematica to find the solutions for

291 equations $(26)-(29)$.

292 2.3.2. Stability

293 Furthermore, we evaluated the stability, by calculating the dominant eigenvalue of the

294 Jacobian matrix for the system of 200 differential equations. At each equilibrium of the 295 system, we compute the Jacobian

$$
\mathbf{J}=\left(\begin{array}{cc}
\mathbf{A}_{1} & \mathbf{B}_{1,2} \\
\mathbf{B}_{2,1} & \mathbf{A}_{2}
\end{array}\right)
$$

296 with:

$$
\mathbf{A}_{i}=\left(\begin{array}{cccc}
\frac{\partial w_{i, 1}}{\partial n_{i, 1}} & \frac{\partial w_{i, 1}}{\partial n_{i, 2}} & \cdots & \frac{\partial w_{i, 1}}{\partial n_{i, 100}} \\
\frac{\partial w_{i, 2}}{\partial n_{i, 1}} & \frac{\partial w_{i, 2}}{\partial n_{i, 2}} & \cdots & \frac{\partial w_{i, 2}}{\partial n_{i, 100}} \\
\vdots & & \ddots & \vdots \\
\frac{\partial w_{i, 100}}{\partial n_{i, 1}} & \frac{\partial w_{i, 100}}{\partial n_{i, 2}} & \cdots & \frac{\partial w_{i, 100}}{\partial n_{i, 100}}
\end{array}\right)
$$


297 and

$$
\mathbf{B}_{i, j}=\left(\begin{array}{cccc}
\frac{\partial w_{i, 1}}{\partial n_{j, 1}} & \frac{\partial w_{i, 1}}{\partial n_{j, 2}} & \cdots & \frac{\partial w_{i, 1}}{\partial n_{j, 100}} \\
\frac{\partial w_{i, 2}}{\partial n_{j, 1}} & \frac{\partial w_{i, 2}}{\partial n_{j, 2}} & \cdots & \frac{\partial w_{i, 2}}{\partial n_{j, 100}} \\
\vdots & & \ddots & \vdots \\
\frac{\partial w_{i, 100}}{\partial n_{j, 1}} & \frac{\partial w_{i, 100}}{\partial n_{j, 2}} & \cdots & \frac{\partial w_{i, 100}}{\partial n_{j, 100}}
\end{array}\right) .
$$
317 feedbacks.

318

When estimating the actual stability of an equilibrium, we set the mutation rate to 0 . The reason is, that with a nonzero mutation rate, the final trait distribution will not be monomorphic at either $z=0$ or $z=1$, due to the selection-mutation balance. However, this slight mismatch may also affect the value of the equilibria. To circumvent this issue, we evaluate the stability in the limit $\mu \rightarrow 0$, where the mutation-selection balance is also expected to be fully favoring selection. Finally, we confirmed the stability metrics using numerical solutions to the system of differential equations, as presented in SI S1.

\subsubsection{Eco-evolutionary model results}

Fig. 7 shows time series generated by the model with traits included. Initially, the populations were monomorphic, with all individuals having a trait value of either $z=0$ or $z=1$. When the initial trait value in the population was $z=0$, both patches reached very similar equilibrium population density, regardless of mutation rate (red and blue lines). Hence, the system allows for stable spatial density variation even without variation in trait value. In contrast, when starting with a monomorphic population with trait value $z=1$, the presence of mutations qualitatively affected the population dynamics (orange and yellow lines). In the absence of mutations, both patches reached the same density (orange line). With mutations, however, the system reached a final equilibrium in which both patches contained a different number of individuals, as well as different trait distributions (yellow lines). In this case the system was thus governed by eco-evolutionary

In the region of parameter space explored here, simultaneous maintenance of variation in abundance and diversification of trait values depends strongly on the values of the parameters $c_{0}$ to $c_{6}$. In Fig. 8, we again varied two parameters at a time while keeping the others constant at the values in Fig. 7. We divided the parameter space into regions with at least one stable equilibrium with variation in both $z$ and $N$, regions with no 
(a)

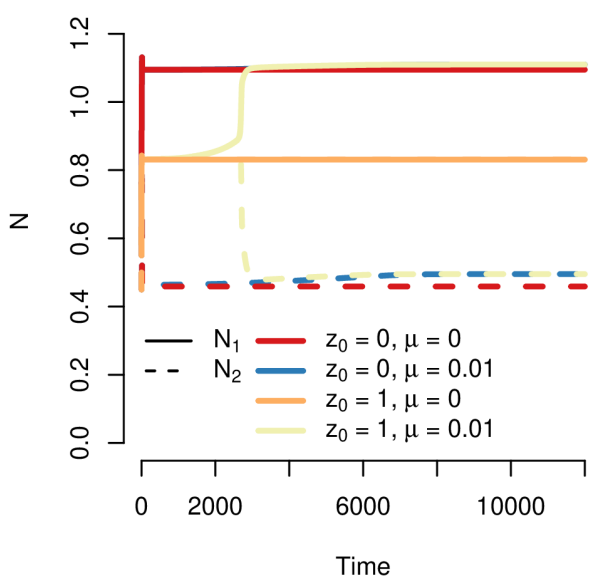

(c)

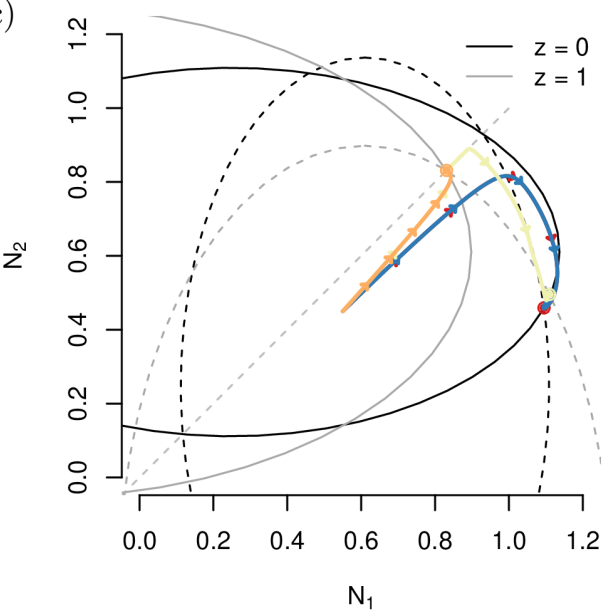

(b)

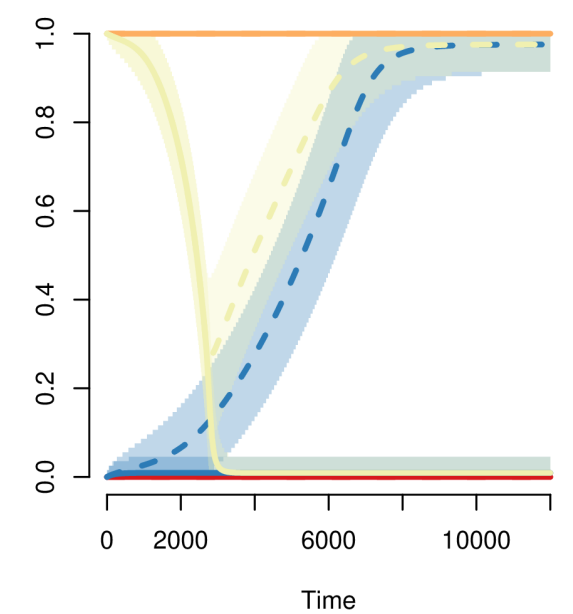

(d)

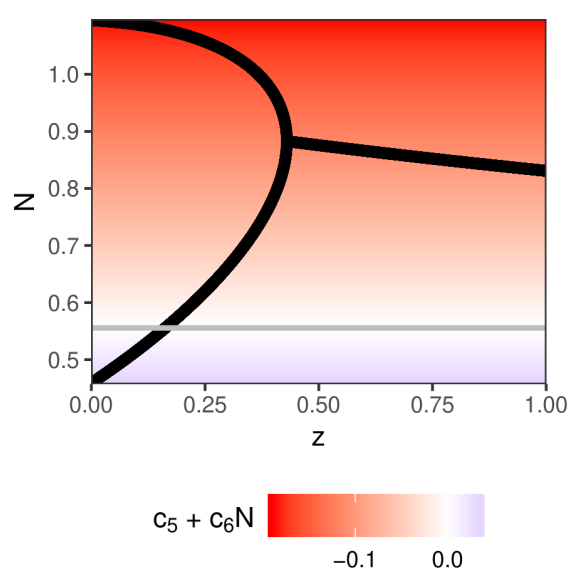

Figure 7: Example time series for the eco-evolutionary model. Different colors represent different model runs where mutations were either present $(\mu=0.01)$ or not $(\mu=0)$ and that started with a monomorphic population with starting trait value $z_{0}$ either 0 or 1. (a) Abundance time series. The red and blue trajectories largely overlap for the first 2000 time steps, as do the yellow and orange lines. (b) Corresponding mean trait values and the spread, shown as the regions in trait space that contained $95 \%$ of the individuals of each patch. For the scenarios without mutations (orange and red line), the trait values in both patches completely overlap. For the scenario with mutations starting at $z=1$ (the yellow lines), initially the lines in both patches overlap, but around time 2500, the trait values in the two patches start to diverge. (c) Trajectories in phase space. Also drawn are the isoclines at $z=0$ (black) and $z=1$ (grey). (d) Equilibrium densities for monomorphic populations with trait value $z$. On the left side of the graph, at any given value of $z$ two branches exist, indicating the two different densities that the two patches will tend to. In the background, the direction of selection at any given density is shown, with red values referring to selection for smaller trait values and blue colors to selection for larger trait values. The grey line corresponds to the density at which selection vanishes. Parameter values: $c_{0}=-0.148, c_{1}=0.162$, $c_{2}=1.262, c_{3}=-0.326, c_{4}=-1.034, c_{5}=0.194$ and $c_{6}=-0.3492$. 
stable joint variability but with the possibility of stable variation in $N$. We also looked

for regions with the possibility of stable variation just in $z$ but did not find any.

Simultaneous trait diversification and variation in abundance exists and depends critically on the ratio between $c_{5}$ and $c_{6}$. As noted above, the selection gradient vanishes when $c_{5}+c_{6} N_{1}=0$. This happens at the critical density $N_{\text {crit }}=-\frac{c_{6}}{c_{5}}$. Above and below this threshold, selection acts in the opposite direction. If one of the two patches of a system is below $N_{\text {crit }}$ and the other above, the trait value will diverge between the patches. In the example shown in Fig. $7, N_{\text {crit }}=0.56$. Given the sign of $c_{5}$ (positive) and $c_{6}$ (negative) that we used, evolution in patches with a density below 0.56 is towards higher values of $z$, while patches with a density above 0.56 tend towards lower values of $z$, as indicated by the grey line and color gradient in Fig. $7(\mathrm{~d})$. However, if $c_{5}$ and $c_{6}$ would have the same sign, $N_{\text {crit }}$ would be negative, and both patches will always have a density higher than $N_{\text {crit }}$. In this case the direction of evolution is density-independent and only depends on the sign of $c_{5}$ and $c_{6}$. This is visible in the top right panel in figure 8 , where all regions of stable trait variation lie in the quadrants where $c_{5}$ and $c_{6}$ have the opposite sign. When altering only $c_{5}$ or $c_{6}$, but not the other, stable coexistence can only occur when the sign of the coefficients does not change (the first five columns of the two top rows in Fig. 8). The importance of the ratio between $c_{5}$ and $c_{6}$ is further stressed in the topright panel in Fig. 8. If the regions with stable variation in both trait value and abundance would be determined by the effect of $c_{5}$ and $c_{6}$ on $N_{\text {crit }}$ only, we would expect the region to be demarcated by two straight lines through the origin in the $c_{5}, c_{6}$-panel. However, the values of $c_{5}$ and $c_{6}$ not only affect $N_{\text {crit }}$, but simultaneously the values of the equilibria, which is why the actual regions for stable variation in trait value and abundance deviate somewhat from the area between the imaginary straight lines through the origin.

Compared to the ecological model, $c_{5}$ and $c_{6}$ introduce a linear trait dependence to $c_{0}$ and $c_{1}$ respectively. In Fig. 8 , this is visible in terms of a strong negative relation between $c_{0}$ and $c_{5}$ as well as $c_{1}$ and $c_{6}$ for cases where long-term variation in $N$ can be maintained, visible as a turquoise diagonal band in the upper left quadrant of the $c_{0}, c_{5}$ and the $c_{1}, c_{6}$ panels. Similarly, also for the region where variation in both $z$ and $N$ can be maintained, larger values of $c_{1}$ allow for more negative values of $c_{6}$. 
bioRxiv preprint doi: https://doi.org/10.1101/759415; this version posted September 8, 2019. The copyright holder for this preprint (which was not certified by peer review) is the author/funder, who has granted bioRxiv a license to display the preprint in perpetuity. It is made available under aCC-BY-ND 4.0 International license.

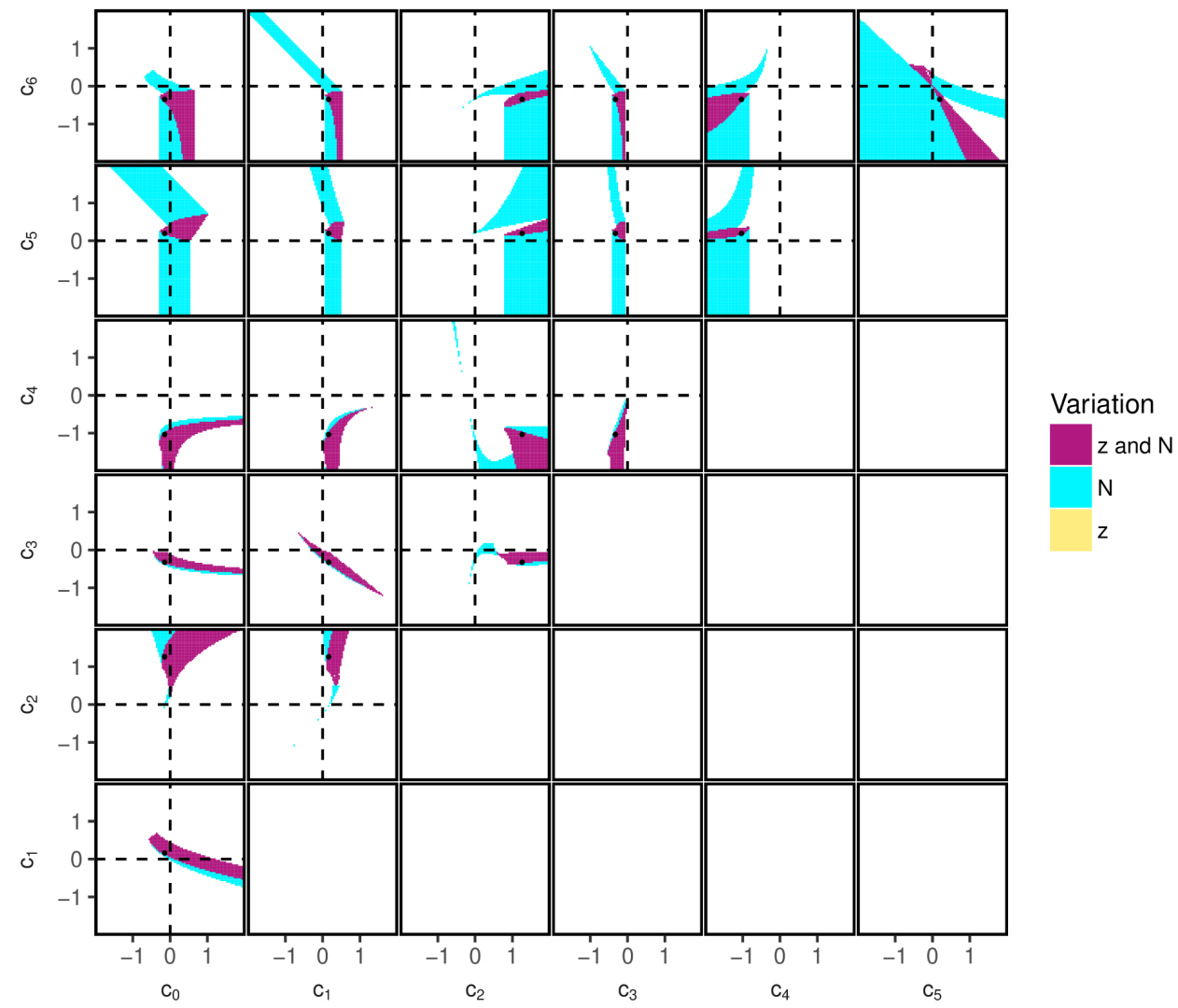

Figure 8: Regions in parameter space where spatial variation in abundance and diversification of trait values can occur. The shown regions are based on the properties of the asymmetric, equilibria obtained by intersecting the ellipses with trait value $z=0$ and $z=1$ with the mirrored ellipses at both trait values (following equations 26) - (29). The values and stability of these ellipses were calculated using Mathematica. Only the upper diagonal graphs are shown. The white regions correspond to areas where no spatial variation in $N$ or $z$ was predicted in the system or where the predicted equilibrium was unstable or unreachable (negative $N$, or a nonzero imaginary part in $N$ ). Note that even in regions with stable density variation and or trait diversification it may depend on the initial conditions whether such an equilibrium is attained or not. The black dots correspond to the parameter settings that were used in the top panels of Fig. 7. In SI S2, the same figure, but including the unstable regions is shown, while in SI S1, a numerical verification of these results is shown. 
353 As for the lower four rows in the graph, the regions where variation in abundance can

354 occur together with trait diversification, are generally a subset of the regions in Fig. 5 where variation in abundance was long-term stable. The values for $c_{0}-c_{4}$ that were used to produce the parameter space figure of the evolutionary model were identical to those used for the parameter space figure of the ecological model. If $z$ were kept to 0 in the evolutionary model, we would retain the ecological model. However, visually, from Fig. 5 and 8 it seems that the possibility of the trait to evolve to a value of 1 , in our specific model, seems to largely divide the regions where stable variation in abundance can occur into those where this can happen together with trait diversification and those without, without changing the general shape of these regions. Furthermore, for our focal parameter combination at least, inclusion of trait evolution produced a few novel regions where stable variation in $N$ can be maintained.

\subsection{Migration}

To explore the robustness of our results to migration between patches, we included migration terms into both the ecological and eco-evolutionary model. We assume that each individual migrates to the respective other patch with a rate $m$, independently of the current population density or the individual's trait value. Detailed methods and results are described in SI S4. In brief, we find that our results on the emergence of spatial density variation and trait diversification are robust to small amounts of migration between patches. With increasing migration rate, spatial heterogeneity decreases and eventually breaks down, first for traits and then for densities. In the example in Fig. 9, the smallest non-zero migration rate leads to both spatial density variation and trait variation, as in the model without migration. An intermediate migration rate still allows for spatial density variation, but trait variation disappears. And for the highest migration rate, both population densities and traits become homogeneous in space.

\subsection{Individual-based models}

In order to test how the results change in the presence of multi-locus genetics, as well as demographic stochasticity, we repeated parts of the analysis with an individual-based 
(a)

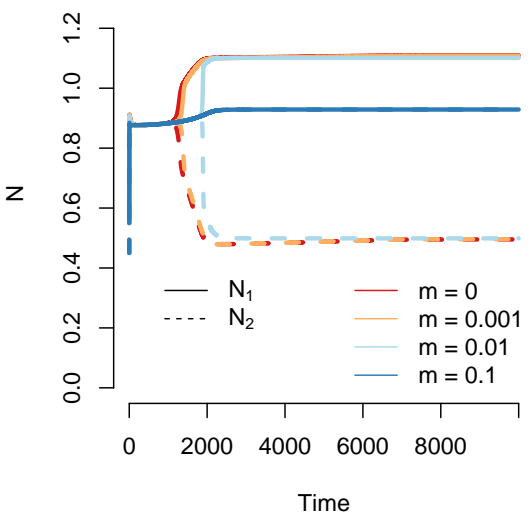

(b)

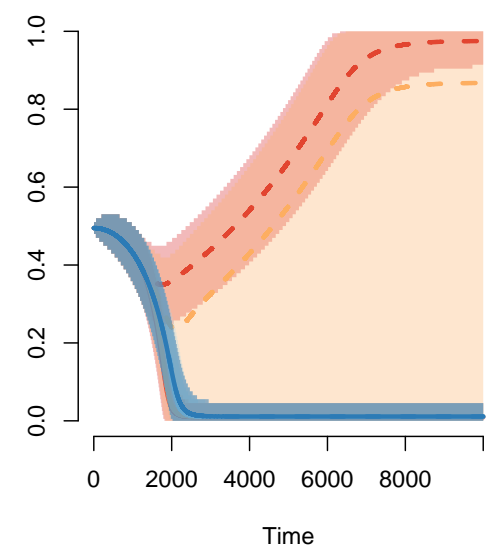

Figure 9: Time series from numerical solutions of the eco-evolutionary model with migration (see S4 for details) and an initial trait value $z_{0}=0.5$. The other parameter values are the same as in Fig. 7 . The shaded regions in panel (b) show the region in trait space that contains $95 \%$ of the population of each patch. For migration rates 0.01 and 0.1 , both patches reach the same final average trait value; these lines completely overlap. When migration is absent, a high average trait value is reached in the low density patch. For $m=0.001$, a high average trait value is still achieved in the low density patch, although the large shaded region implies that the patch also contains a non-negligible fraction of low-trait value individuals. With increasing migration rates, first the adaptation disappears (light blue lines and shaded regions overlapping with the blue line and region in panel b) and when $m=0.1$ even the difference in abundance between the patches disappears. 
model (IBM, see SI S5 for details). With appropriate parameter choices, spatial density variation and trait diversification did occur in the individual-based model. An additional parameter in the IBM was patch area. With small patch area, patches could accommodate few individuals and demographic stochasticity was strong, leading to frequent extinctions. With large patch area, the number of individuals was larger in total, there was less demographic stochasticity and the results were more similar compared to the deterministic model.

\section{Discussion}

In this study, we have explored the ecological and eco-evolutionary consequences of multiscale density dependence where an individual's fitness depends not just on population density in its own patch but also on the density in another patch in the region. We have shown that multi-scale density dependence may lead to the emergence and stable maintenance of spatial variation in population densities in an otherwise homogeneous environment and to the diversification of traits under density-dependent selection. That is, without any extrinsic heterogeneity, different niches emerged in the population, with some individuals being better adapted to low-density situations and others to higher density. We also observed how spatial density patterns and trait variation influenced each other through eco-evolutionary feedbacks. Specifically, we have shown a case where spatial density variation arose only when traits were allowed to evolve. Our model thus emphasizes how eco-evolutionary feedbacks can qualitatively affect both trait and population dynamics.

\subsection{Formation of density patterns in space}

In nature, spatial variation in population density between patches or subpopulations of the same species is ubiquitous. Our study highlights one possible mechanism that can produce or contribute to spatial density variation. A variety of other mechanisms exist. Firstly, evidence from natural populations suggests, that abiotic or biotic environmental conditions, such as temperature, climatic stability, precipitation, or food availability are key drivers of variation in density (Santini et al., 2018). Observed spatial variation in 
density can, however, also simply be caused by stochasticity, although such variation will not be stable over longer time scales. Finally, there are explanations that require neither extrinsic heterogeneity, nor stochasticity. Our study falls in this last category.

Many models from this last category (e.g. Bolker and Pacala, 1997; Bolker, 2003; Sasaki, 1997) focus on the interplay between random, undirected dispersal in some local neighbourhood, and intraspecific competition with other individuals in some competition neighbourhood. If typical dispersal distances are small relative to the spatial scale of competition, clusters of high population density can form. Similar to our model, these models also require some degree of non-local competition for pattern formation. Stable density variation can also emerge as the product of dispersal directed towards high population density (see e.g. Ellis et al., 2019), the interplay between small-range facilitation and long-range resource competition (van de Koppel et al., 2005), interactions with other species, such as reproductive interference (Ruokolainen and Hanski, 2016), hostparasitoid or -parasite interactions (Boots and Sasaki, 2000; Hassell et al., 1994), and interspecific competition acting over a smaller spatial scale than intraspecific competition (Murrell and Law, 2003).

Other studies have considered Allee effects as a key ingredient for the emergence and maintenance of spatial heterogeneity. For example, Gyllenberg and Hemminki (1999) showed how Allee effects caused by mate-finding difficulties together with non-local competition can lead to stable density differences between patches, even with nonzero migration. Their model is similar to our ecological model, except we did not explicitly model a specific Allee effect. Another study has shown how Allee effects can cause a population to be completely absent from some areas while being present in others, thereby limiting the spread of an invasive species (Keitt et al., 2001). Spatial heterogeneity in the sense of presence-absence variation can also be explained by metapopulation models (as first developed by Levins, 1969), but since each patch in a metapopulation experiences recurrent extinction and recolonization events, density patterns will not be stable over time.

More generally, all population models with alternative stable states can generate stable spatial heterogeneity in population density under appropriate initial conditions and with sufficiently small migration between locations. But without feedbacks between the 
439 patches, i.e. multi-scale density dependence, symmetric situations would always be as

440 stable as asymmetric situations. Our model allows for situations where asymmetric den-

441 sities are the only stable equilibria at which the species can exist (see for example Fig. 4

442 top, Fig. 6).

444 In our eco-evolutionary model, a necessary but not sufficient condition for the mainte-

\subsection{Maintenance of trait variation and relation to other coexistence mechanisms}

445 nance of trait variation is the simultaneous maintenance of spatial variation in population density (see Fig. 8). Under appropriate parameter combinations, stable trait variation emerges with a high-density specialist dominating in the high-density patch and a lowdensity specialist dominating in the low-density patch, essentially a case of local adaptation (see Fig. 7). At equilibrium, there is still a very small amount of within-patch trait variation around the optimal trait value due to mutation-selection balance.

Chesson's coexistence theory is a powerful framework to understand and classify coexistence mechanisms under spatio-temporal heterogeneity (Chesson, 2000). Yet, the coexistence between a high-density specialist and a low-density specialist in our model does not appear to fit straightforwardly into this framework. Because we have two types and two limiting factors, e.g. the densities in the two patches or functions of them, we do not seem have one of the cases where invasion growth rates can be cleanly partitioned into contributions from fluctuation independent frequency-dependence, storage effects, relative nonlinearity etc. (Barabás et al., 2018). Instead, we here provide an intuitive reasoning for how mutual invasibility and coexistence are achieved in our model.

Mutual invasibility and therefore stable coexistence of a high-density specialist and a low-density specialist can be achieved in two ways in our eco-evolutionary model. The first scenario is that each strategy produces spatial variation in density on its own and the high-density specialist can invade the high-density patch of the low-density specialist and the low-density specialist can invade the low-density patch of the high-density specialist. This scenario is illustrated by the example in Fig. S3.1. The second scenario is that the low-density specialist on its own has the same density in both patches, a density that allows the high-density specialist to invade, and the high-density specialist produces 
493 As described in section 3.1 and SI S4, migration (and dispersal) can have profound

spatial variation in density such that the low-density specialist can invade the less dense patch. This is the scenario in Fig. 7. If neither the high-density specialist nor the lowdensity specialist have spatial variation in density on their own, mutual invasibility does not appear possible in our model.

To our knowledge, the role of multi-scale density-dependent selection in the maintenance of polymorphism has not been investigated before. Engen and Sæther (2019), however, showed that density-dependent selection can affect spatial trait patterns in a model with dispersal and temporal environmental heterogeneity. Also, there is previous work on how the spatial scale of density regulation in patchy landscapes affects the maintenance of polymorphism (Ravigné et al., 2004). If density regulation happens locally after selection, this is called soft selection (Levene model, Levene, 1953). If density regulation happens globally, this is called hard selection (Dempster model, Dempster, 1955). In soft-selection models, population densities are usually not affected by selection or migration, whereas in hard-selection models they may be affected (Lenormand, 2002). It has been shown that compared to hard selection, soft selection is more conducive to maintenance of polymorphism in response to environmental heterogeneity (e.g. Ravigné et al., 2004). More recently, also mixtures of hard and soft selection have received attention (De Meeûs and Goudet, 2000; Débarre and Gandon, 2011). The environmental heterogeneity in these studies, however, was assumed to be unrelated to population density. In our model, selection and density-regulation cannot be clearly separated and thus our model does not fit perfectly into the hard-selection/soft-selection framework. It shares more aspects with hard-selection models, most importantly that fitness influences absolute number of offspring and population densities, but there are also many additional aspects in our model like Allee effects and feedbacks between density and selection.

\subsection{Migration and stochasticity}

effects on the maintenance of variation in abundance. Like our ecological model, our evolutionary model is also robust against small amounts of migration (Fig. 9 and SI S4). However, with increasing migration rates, the accompanying gene flow between the patches hampers adaptation through gene swamping (Lenormand, 2002). This effect is 
524

expected to be particularly strong when the difference in abundance between the two patches is large, and a disproportionately large number of individuals migrate from the larger to the smaller patch. In a system where the patches have more similar abundances, gene swamping is thus expected to have a smaller effect, although the evolution of densitydependent traits would also be slower due to a weaker selection gradient. At higher migration rates, migration hampers not only adaptation, but even leads to the system reaching an equilibrium in which both patches have the same abundance. We thus only see negative effects of migration on long-term differences in abundances and trait values between the two patches in the deterministic models.

Several pathways exist through which migration (or dispersal) can have positive effects on local adaptation. Specifically for evolution, trait-dependent migration can lead to trait diversification. Homogeneous migration may also aid adaptation, for example by resupplying alleles that have been lost to drift (Blanquart et al., 2012), or by preventing a patch from going extinct, leading to more time for an adaptation to spread (Gomulkiewicz et al., 1999). In our deterministic model, these effects do not play a role because migration is unstructured, and alleles can always re-emerge. When demographic stochasticity is included, as in our individual based model (SI S5), migration can have positive effects on diversification. In those model runs, migration counteracted stochastic extinctions of the smaller patch for long enough, such that adaptation to low density could take place, similar to the effect described by Gomulkiewicz et al. (1999). This effect disappears when the absolute number of individuals increases, thereby weakening the effect of demographic stochasticity on population level processes.

Stochasticity may also promote trait diversification regardless of migration. Due to the symmetry in the deterministic model, if the two patches have the exact same initial abundance and trait distribution, they cannot diverge over time. In such cases, small amounts of (demographic) stochasticity may lead to small differences between the patches that are then amplified by the internal model dynamics, leading to variation in abundance 25 as well as trait diversification. 
555

\subsection{Interpretation and applications}

Our model assumes that density dependence plays out on more than one spatial scale. Such multi-scale density dependence should be common because an individual's fitness depends on many different processes and factors, such as juvenile survival, protection from disturbances, resource competition, mate finding, competition for mating partners, reproduction, interactions with other species etc., which will generally occur over different spatial scales (see e.g., Cook et al., 2001; Gascoigne et al., 2005; Rietkerk, 2004). However, not all forms of multi-scale density dependence will lead to spatial variation in population densities and trait diversification. In our model, when there is positive density dependence at low density at both scales, spatial density variation can only be stable if the positive effects of conspecifics at low density and the negative effects at high density are either both strongest for the own patch or both strongest for the other patch. However, even then, not all parameter choices lead to stable density variation (compare Fig. 4(a) to (c)). Moreover, in almost all our examples with stable variation $c_{1}<c_{2}$ with a positive $c_{2}$ and $c_{1}$ being either negative or positive, suggesting that facilitation from the other patch is more important for density and trait variation than facilitation by individuals in the same patch. However, we can currently not generalize these claims for all of parameter space.

The requirements for stable density variation and trait diversification could be fulfilled for example in plant-pollinator systems where plants grow in two patches but pollinators are more mobile and can visit both patches. A focal plant patch may benefit from a small nearby patch, by guiding pollinators to the focal patch. However, when density in the nearby patch gets too large, pollinators may instead choose to spend most of their time at that nearby patch. Simultaneously, within the focal patch, high density may lead to higher resource competition, while low densities may make the patch difficult to find

1 for pollinators. When these processes lead to asymmetric abundances across the patches, this in turn affects the optimal investment that plants should make in competitive ability. This long-term difference can then lead to the emergence of trait variation. Evolutionary processes can also affect the abundances of the system, and can lead to a shift from a 5 symmetric to an asymmetric equilibrium in abundances and subsequently to adaptation in density. 
The patches in our model can also be interpreted in terms of social groups rather than locations, such as bark beetles attacking a tree (Raffa et al., 2008), or cooperative breeders. Similarly, the two groups can also be interpreted in terms of two competing (identical) species in a single patch, as modelled by Gerla and Mooij (2014). Although we had originally not thought of this interpretation, it turns out that mathematically, the model described by Gerla and Mooij (2014) is nearly equivalent to the ecological version of our model without evolution and migration. They find the same equilibria and shapes of the isoclines, but do not fully assess the stability of the asymmetric equilibria. Interestingly, Gerla and Mooij (2014) also mention plant-pollinator dynamics as a biological example, noting specifically the similarities between their system and the plant-pollinator model by Lutscher and Iljon (2013). A similar interpretation could be applied to our study as noted above. However, our study differs by focusing on spatial dynamics, deriving a direct equation for the stability of the unstable equilibrium, and by also evaluating the effects of trait evolution, migration and stochasticity.

The actual occurrence of the here-described eco-evolutionary effects require empirical evidence. It is currently unclear whether the sets of coefficients that allow for spatial density variation and trait diversification occur in natural populations. The fitness coefficients could empirically be estimated by evaluating how the fitness in one patch varies when its density and the density of a nearby patch are manipulated. Alternatively, one could experimentally test predictions of our model. In our model, if an asymmetric equilibrium with one patch at high density and one patch at low density is stable, the mirrored situation with the respective other patch at high or low density should also be stable. Our model thus predicts that if one manipulated a system with asymmetric densities, for example by adding individuals to one patch and removing individuals from the other patch, the system should eventually shift from the basin of attraction of one asymmetric equilibrium to the other asymmetric equilibrium and stay there. By contrast, if the spatial density variation is due to extrinsic environmental differences, the system should return to the original equilibrium. Once it has been established that the system is ecologically capable of reaching asymmetric abundances, experimental evolution may be attempted. Although such experiments are challenging, testing the effects of eco-evolutionary dynamics can and has been done, for example by frequently replacing individuals in an 
experimental population by wildtype individuals from a stock population, thereby disabling the evolutionary part of the feedback loop and comparing such a population to a population where evolution is allowed to take place (e.g. Hart et al., 2019).

\subsection{Limitations and future work}

We assumed a homogeneous environment, which may be unrealistic for most natural populations. Whether the effects described here are of importance when the environment is heterogeneous remains to be investigated. This could be evaluated through a model that also incorporates environmental heterogeneity for example through an additional term in the fitness function. Depending on the spatio-temporal pattern of environmental variation, many different model behaviors may be possible. We expect, however, that our results will be robust to some degree of environmental heterogeneity. Even if the patches are slightly different in their properties, the eco-evolutionary feedbacks described here should still act and while it might be more likely for the patch with slightly better environmental conditions to become the high density patch, depending on the starting conditions it may also be the other way around if the eco-evolutionary feedbacks are strong enough. The phenomena described here could also amplify the effects of environmental heterogeneity on spatial density variation. This interplay between intrinsic and extrinsic factors for the maintenance of eco-evolutionary variation is a promising direction for future research.

Temporally strongly varying environments may favor plastic rather than evolutionary responses: phenotypic plasticity could allow an individual to cope well with the different environments that it will experience over its life time. In our model, the environments that individuals and their offspring encounter critically depend on the migration rate and we would expect phenotypic plasticity to be favorable when the migration rate is small enough for spatial density variation to persist, but too large for evolutionary trait diversification. Generally, whether plasticity can evolve depends on the costs and limits of the plasticity (DeWitt et al., 1998), such as the degree of unpredictability of the future environment (Reed et al., 2010), in conjunction with the migration rate and 6 the accuracy of the plastic response (Sultan and Spencer, 2002). The precise conditions 7 should be explored in future extensions of our model. 
Another potential key factor that determines the model behaviour is the spatial structure. We have investigated a model with a particular, very simple spatial set-up: two patches that mutually affect each other. An important direction for future work is to investigate how the eco-evolutionary dynamics explored in this study play out in other spatial settings, for example landscapes with more than two patches, or continuous space.

In such scenarios, individuals could respond to spatial density variation in multiple ways, e.g. to the density in their own patch or neighborhood and to the density in the rest of the landscape, or separately to the densities in each patch, or as defined by a kernel based on distance. In continuous-space models, local dispersal is another mechanism that could interact with multi-scale density dependence to promote spatial variation in density. We speculate that at larger scales too, multi-scale density dependence may promote density and trait variation, although this needs to be confirmed with additional models.

Individuals in our model differ in just one trait and the fitness effects of this trait depend on just the population density in the individual's own patch, even though fitness itself is affected by density on multiple scales. Future models could incorporate traits that are involved in density-dependent processes at a larger scale and whose fitness effects therefore depend on the density in the other patch or on total density. We speculate that similar eco-evolutionary feedbacks as we reported here would act in this case, which should also allow for the maintenance of trait variation under appropriate conditions.

Although much remains to be investigated, we argue that multi-scale density dependence is a common, potentially very important phenomenon in evolutionary ecology. Based on many empirical examples (see e.g., Cook et al., 2001; Gascoigne et al., 2005; Rietkerk, 2004) and the argument that fitness depends on multiple biological processes that will generally not play out at exactly the same spatial scale, we expect that multi-scale density dependence will be the rule rather than the exception. More generally, when studying natural systems, the outcome may depend on the, sometimes arbitrarily, chosen spatial scale over which the study is conducted (Kareiva, 1990; Murphy, 1989; Ray and Hastings, 1996; Snyder and Chesson, 2004) and multi-scale density dependence could contribute to explaining some of these inconsistent results. Finding relevant literature and synthesizing information on multi-scale density dependence is, however, made difficult by the lack of clear terminology for this phenomenon. Because of its important ecological and eco- 
649 evolutionary consequences which we highlighted in this study, we argue that multi-scale density dependence should receive more concentrated research attention.

\section{Author contributions}

664

\section{Conclusion}

\section{Acknowledgements} interpretation of the results.

This research was funded by the German Research Foundation (DFG) as part of the SFB TRR $212\left(\mathrm{NC}^{3}\right)$. Furthermore we are grateful to Hannah Živković and Matthias Kubacki for their contributions to the individual based model.

$\mathrm{KvB}$ and MW conceived the ideas and designed the methodology. KvB led the analyses and the writing of the manuscript. MW contributed extensively to the writing and

\section{References}

Barabás, G., D'Andrea, R., Stump, S. M., 2018. Chesson's coexistence theory. Ecological Monographs 88 (3), 277-303.

Berec, L., Kramer, A. M., Bernhauerová, V., Drake, J. M., 2018. Density-dependent selection on mate search and evolution of Allee effects. Journal of Animal Ecology 87 (1), 24-35.

Blanquart, F., Gandon, S., Nuismer, S., 2012. The effects of migration and drift on local adaptation to a heterogeneous environment. Journal of Evolutionary Biology 25 (7), 1351-1363.
} 
Bolker, B., Pacala, S. W., 1997. Using moment equations to understand stochastically driven spatial pattern formation in ecological systems. Theoretical Population Biology 52 (3), 179-197.

Bolker, B. M., 2003. Combining endogenous and exogenous spatial variability in analytical population models. Theoretical Population Biology 64 (3), 255-270.

Boots, M., Sasaki, A., 2000. The evolutionary dynamics of local infection and global reproduction in host-parasite interactions. Ecology Letters 3 (3), 181-185.

Chesson, P., 2000. Mechanisms of maintenance of species diversity. Annual Review of Ecology and Systematics 31 (1), 343-366.

Cook, W. M., Holt, R. D., Yao, J., 2001. Spatial variability in oviposition damage by periodical cicadas in a fragmented landscape. Oecologia 127 (1), 51-61.

Courchamp, F., Berec, L., Gascoigne, J., 2008. Allee effects in ecology and conservation. Oxford University Press.

De Meeûs, T., Goudet, J., 2000. Adaptive diversity in heterogeneous environments for populations regulated by a mixture of soft and hard selection. Evolutionary Ecology Research 2 (8), 981-995.

Débarre, F., Gandon, S., 2011. Evolution in heterogeneous environments: between soft and hard selection. The American Naturalist 177 (3), E84-E97.

Dempster, E. R., 1955. Maintenance of genetic heterogeneity. Cold Spring Harbor Symposia on Quantitative Biology 20, 25-32.

DeWitt, T. J., Sih, A., Wilson, D. S., 1998. Costs and limits of phenotypic plasticity. Trends in Ecology \& Evolution 13 (2), 77-81.

Ellis, J., Petrovskaya, N., Petrovskii, S., 2019. Effect of density-dependent individual movement on emerging spatial population distribution: Brownian motion vs Levy flights. Journal of Theoretical Biology 464, 159-178.

Engen, S., Sæther, B.-E., 2019. Ecological dynamics and large scale phenotypic differentiation in densitydependent populations. Theoretical Population Biology 127, 133-143.

Gage, M. J., 1995. Continuous variation in reproductive strategy as an adaptive response to population density in the moth Plodia interpunctella. Proceedings of the Royal Society of London. Series B: Biological Sciences 261 (1360), 25-30.

Gascoigne, J., Berec, L., Gregory, S., Courchamp, F., 2009. Dangerously few liaisons: a review of matefinding Allee effects. Population Ecology 51 (3), 355-372.

Gascoigne, J. C., Beadman, H. A., Saurel, C., Kaiser, M. J., 2005. Density dependence, spatial scale and patterning in sessile biota. Oecologia 145 (3), 371-381.

Gerla, D. J., Mooij, W. M., 2014. Alternative stable states and alternative endstates of community assembly through intra-and interspecific positive and negative interactions. Theoretical Population Biology 96, 8-18.

Gomulkiewicz, R., Holt, R. D., Barfield, M., 1999. The effects of density dependence and immigration on local adaptation and niche evolution in a black-hole sink environment. Theoretical Population Biology 55 (3), 283-296.

Govaert, L., Fronhofer, E. A., Lion, S., Eizaguirre, C., Bonte, D., Egas, M., Hendry, A. P., De Brito Mar- 
tins, A., Melián, C. J., Raeymaekers, J. A., et al., 2019. Eco-evolutionary feedbacks-theoretical models and perspectives. Functional Ecology 33 (1), 13-30.

Gyllenberg, M., Hemminki, J., 1999. Allee effects can both conserve and create spatial heterogeneity in population densities. Theoretical Population Biology 56 (3), 231-242.

Hart, S. P., Turcotte, M. M., Levine, J. M., 2019. Effects of rapid evolution on species coexistence. Proceedings of the National Academy of Sciences 116 (6), 2112-2117.

Hassell, M. P., Comins, H. N., May, R. M., 1994. Species coexistence and self-organizing spatial dynamics. Nature 370 (6487), 290-292.

Kareiva, P., 1990. Population dynamics in spatially complex environments: theory and data. Philosophical Transactions of the Royal Society of London. Series B: Biological Sciences 330 (1257), 175-190.

Keitt, T. H., Lewis, M. A., Holt, R. D., 2001. Allee effects, invasion pinning, and species' borders. The American Naturalist 157, 203-216.

Kokko, H., López-Sepulcre, A., 2007. The ecogenetic link between demography and evolution: can we bridge the gap between theory and data? Ecology Letters 10 (9), 773-782.

Kokko, H., Rankin, D. J., 2006. Lonely hearts or sex in the city? Density-dependent effects in mating systems. Philosophical Transactions of the Royal Society B: Biological Sciences 361 (1466), 319-334.

Lenormand, T., 2002. Gene flow and the limits to natural selection. Trends in Ecology \& Evolution 17 (4), 183-189.

Levene, H., 1953. Genetic equilibrium when more than one ecological niche is available. The American Naturalist 87 (836), 331-333.

Levins, R., 1969. Some demographic and genetic consequences of environmental heterogeneity for biological control. American Entomologist 15 (3), 237-240.

Lutscher, F., Iljon, T., 2013. Competition, facilitation and the Allee effect. Oikos 122 (4), 621-631.

MacArthur, R. H., Wilson, E. O., 1967. The theory of island biogeography. Princeton University Press.

Mueller, L. D., 1997. Theoretical and empirical examination of density-dependent selection. Annual Review of Ecology and Systematics 28 (1), 269-288.

Mueller, L. D., Guo, P., Ayala, F. J., 1991. Density-dependent natural selection and trade-offs in life history traits. Science 253 (5018), 433-435.

Murphy, D. D., 1989. Conservation and confusion: wrong species, wrong scale, wrong conclusions. Conservation Biology 3 (1), 82-84.

Murrell, D. J., Law, R., 2003. Heteromyopia and the spatial coexistence of similar competitors. Ecology Letters 6 (1), 48-59.

Nicholson, A. J., 1957. The self-adjustment of populations to change. In: Cold Spring Harbor Symposia on Quantitative Biology. Vol. 22. Cold Spring Harbor Laboratory Press, pp. 153-173.

Nicolaus, M., Tinbergen, J. M., Ubels, R., Both, C., Dingemanse, N. J., 2016. Density fluctuations represent a key process maintaining personality variation in a wild passerine bird. Ecology Letters 19 (4), 478-486.

Patterson, J. E., Ruckstuhl, K. E., 2013. Parasite infection and host group size: a meta-analytical review. Parasitology 140 (7), 803-813. 
R Core Team, 2018. R: A Language and Environment for Statistical Computing. R Foundation for Statistical Computing, Vienna, Austria.

Raffa, K. F., Aukema, B. H., Bentz, B. J., Carroll, A. L., Hicke, J. A., Turner, M. G., Romme, W. H., 2008. Cross-scale drivers of natural disturbances prone to anthropogenic amplification: the dynamics of bark beetle eruptions. Bioscience 58 (6), 501-517.

Ravigné, V., Olivieri, I., Dieckmann, U., 2004. Implications of habitat choice for protected polymorphysms.

Ray, C., Hastings, A., 1996. Density dependence: Are we searching at the wrong spatial scale? The Journal of Animal Ecology 65 (5), 556.

Reed, T. E., Waples, R. S., Schindler, D. E., Hard, J. J., Kinnison, M. T., 2010. Phenotypic plasticity and population viability: the importance of environmental predictability. Proceedings of the Royal Society B: Biological Sciences 277 (1699), 3391-3400.

Reinhold, K., 2003. Influence of male relatedness on lethal combat in fig wasps: a theoretical analysis. Proceedings of the Royal Society of London B: Biological Sciences 270 (1520), 1171-1175.

Richter-Gebert, J., 2011. Perspectives on projective geometry: A guided tour through real and complex geometry. Springer Science \& Business Media.

Rietkerk, M., 2004. Self-organized patchiness and catastrophic shifts in ecosystems. Science 305 (5692), 1926-1929.

Rodenhouse, N. L., Sillett, T. S., Doran, P. J., Holmes, R. T., 2003. Multiple density-dependence mechanisms regulate a migratory bird population during the breeding season. Proceedings of the Royal Society B: Biological Sciences 270 (1529), 2105-2110.

Ruokolainen, L., Hanski, I., 2016. Stable coexistence of ecologically identical species: conspecific aggregation via reproductive interference. Journal of Animal Ecology 85 (3), 638-647.

Santini, L., Isaac, N. J., Maiorano, L., Ficetola, G. F., Huijbregts, M. A., Carbone, C., Thuiller, W., 2018. Global drivers of population density in terrestrial vertebrates. Global Ecology and Biogeography 27 (8), 968-979.

Sasaki, A., 1997. Clumped distribution by neighbourhood competition. Journal of Theoretical Biology 186 (4), 415-430.

Snyder, R. E., Chesson, P., 2004. How the spatial scales of dispersal, competition, and environmental heterogeneity interact to affect coexistence. The American Naturalist 164 (5), 633-650.

Soetaert, K., Petzoldt, T., Setzer, R. W., 2010. Solving differential equations in R: Package deSolve. Journal of Statistical Software 33 (9), 1-25.

Sultan, S. E., Spencer, H. G., 2002. Metapopulation structure favors plasticity over local adaptation. The American Naturalist 160 (2), 271-283.

Travis, J., Leips, J., Rodd, F. H., 2013. Evolution in population parameters: density-dependent selection or density-dependent fitness? The American Naturalist 181 (S1), S9-S20.

van de Koppel, J., Rietkerk, M., Dankers, N., Herman, P. M. J., 2005. Scale-dependent feedback and regular spatial patterns in young mussel beds. The American Naturalist 165 (3), E66-E77.

Wang, Y., DeAngelis, D. L., 2019. Energetic constraints and the paradox of a diffusing population in a 


\section{Supporting information}

\section{S1. Numerical verification of the parameter space of the evolutionary model}

The results in Fig. 8 were based on the Jacobian and the equilibrium values returned by Mathematica. However, these results are subject to the numerical precision of the computer, which is why we assumed imaginary parts of the equilibria smaller than $10^{-16}$ to be equal to zero and hence as reachable equilibria. We therefore numerically verified the results from Fig. 8 using the package deSolve (Soetaert et al., 2010).

\section{S1.1. Methods}

Because the numerical solutions run slower compared to the analytical results, we performed these at a lower resolution, by dividing the coefficient range $(-2-+2)$ in 20 steps only. Hence every subpanel consists of $20 \times 20$ pixels (and checks). For every parameter setting (i.e. pixel), we first calculated the six possible nonzero equilibria that allow for variation in at least either trait value or abundance, using the analytical solution. These were the four possible mutual intersections of the ellipse corresponding to trait value 0 and trait value 1 , and the asymmetric equilibria for a system where trait values were the same in both patches (either both at 1 or both at 0 , considering only one of the mirrored asymmetric equilibria in each case). Then, we removed the imaginary parts of these results. Furthermore, we remove the equilibria with a negative real part, since these are not biologically relevant.

Subsequently, we evaluated whether the remaining equilibria were stable. For each of the equilibria $\left(N_{1}^{*}, N_{2}^{*}\right)$, we pick four possible starting densities, that differ from the expected equilibrium by $2.5 \%$. Each patch can be either $2.5 \%$ higher or lower, leaving us with the four possible starting conditions: $\left(0.975 N_{1}^{*}, 0.975 N_{2}^{*}\right),\left(0.975 N_{1}^{*}, 1.025 N_{2}^{*}\right),\left(1.025 N_{1}^{*}, 0.975 N_{2}^{*}\right)$, and $\left(1.025 N_{1}^{*}, 1.025 N_{2}^{*}\right)$. We call an equilibrium stable with respect to the density, if for each starting condition, after 100 time steps the difference between the abundance in the patches and their expected value according to the equilibrium is smaller than $2.5 \%$. 
818 In order to make sure that the population goes to the expected equilibrium, we had

$\mathbf{8 1 9}$ to set $\mu=0$, because when mutations are present, the trait values will deviate from

820 0 or 1 by a small amount, due to the mutation-drift balance and this deviation in $z$

821 may subsequently lead to a small difference in abundances as well. Thereby hindering a

$\mathbf{8 2 2}$ direct comparison between the numerically predicted density and the expected density.

${ }_{\mathbf{8 2 3}}$ However, this means that we have to assess the stability of the equilibrium with respect

$\mathbf{8 2 4}$ to $z$ separately. We did so, by changing the initial trait distribution. Instead of putting

825 the full population in either the bin with $z=0$ or $z=1$, we put $99 \%$ of the population

$\mathbf{8 2 6}$ in the extreme bin and $1 \%$ in the adjacent bin. When $z=0$, this corresponds to:

$$
\begin{aligned}
& n_{1,1}(0)=0.99 N_{1}(0) \\
& n_{1,2}(0)=0.01 N_{1}(0) \\
& n_{2,1}(0)=0.99 N_{2}(0) \\
& n_{2,2}(0)=0.99 N_{2}(0),
\end{aligned}
$$

${ }_{\mathbf{8 2 7}}$ and zero density in all other bins. When $z=1$, bins 100 and 99 take the role of bin 1

${ }_{\mathbf{8 2 8}}$ and 2 respectively. If the relative number of individuals in the extreme bin had increased

829 at $t=100$ compared to the initial value, the system was considered to be stable with

$\mathbf{8 3 0}$ respect to trait value.

${ }_{831}$ S1.2. Results

832 The resulting figure (Fig. S1.1) confirms the findings from Fig. 8. 
bioRxiv preprint doi: https://doi.org/10.1101/759415; this version posted September 8, 2019. The copyright holder for this preprint (which was not certified by peer review) is the author/funder, who has granted bioRxiv a license to display the preprint in perpetuity. It is made available under aCC-BY-ND 4.0 International license.

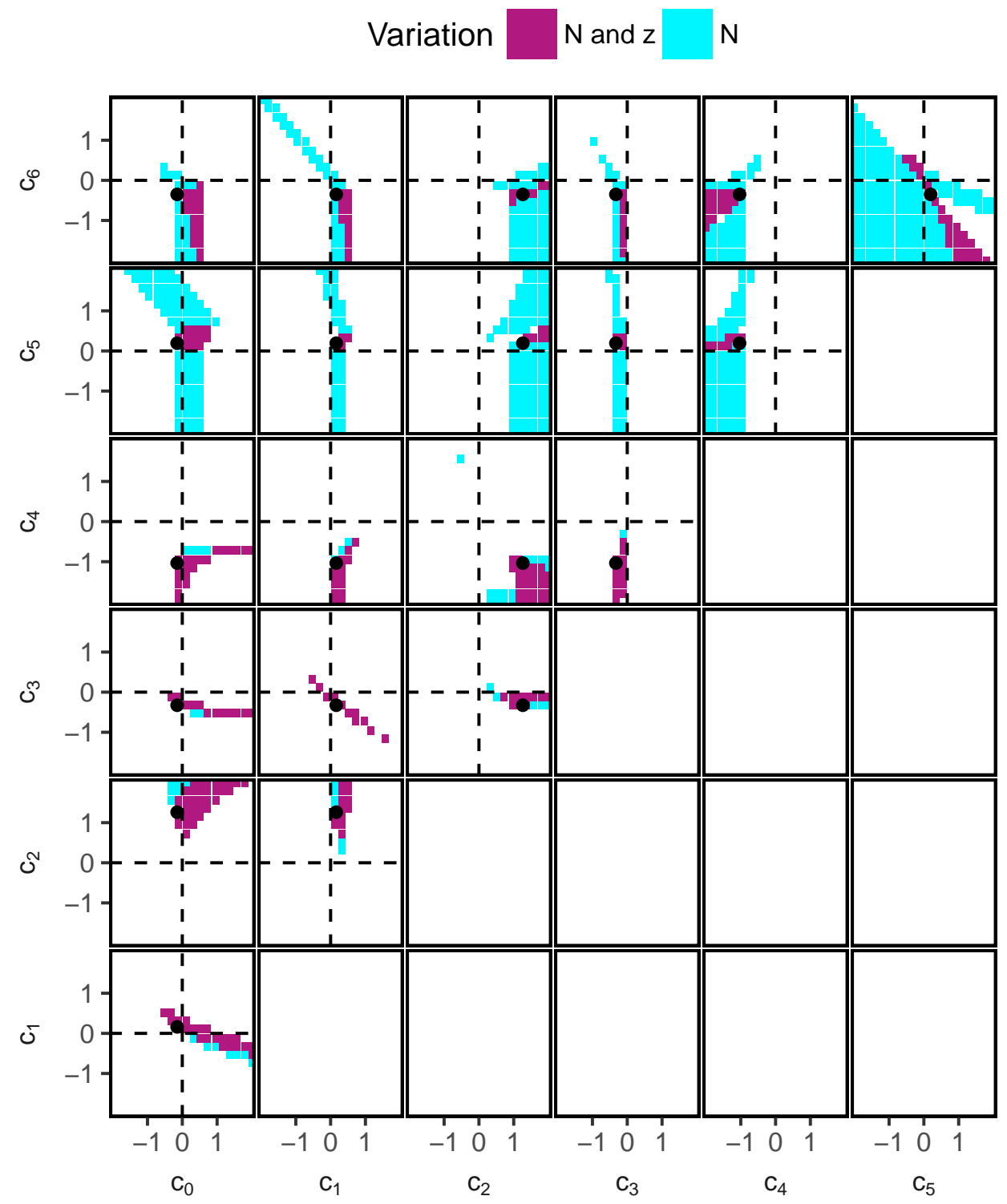

Figure S1.1: Regions in parameter space where spatial variation abundance and diversification of trait values can occur. The shown regions are based on numerically evaluating the population dynamics around the the asymmetric, equilibria that were obtained by intersecting the ellipses with trait value $z=0$ and $z=1$ with the mirrored ellipses at both trait values (following equations 26) - (29). The white regions correspond to areas where no spatial variation in $N$ or $z$ was predicted in the system or where the predicted equilibrium was unstable or unreachable (negative $N$, or a nonzero imaginary part in $N$ ). The black dots correspond to the parameter settings that were used in the top panels of Fig. 7 . The results in thsi graph numerically confirm the findings from Fig. 8. 
bioRxiv preprint doi: https://doi.org/10.1101/759415; this version posted September 8,2019 . The copyright holder for this preprint (which was not certified by peer review) is the author/funder, who has granted bioRxiv a license to display the preprint in perpetuity. It is made available under aCC-BY-ND 4.0 International license.

833 S2. Parameter space of the eco-evolutionary model including unstable equi$834 \quad$ libria 
bioRxiv preprint doi: https://doi.org/10.1101/759415; this version posted September 8, 2019. The copyright holder for this preprint (which was not certified by peer review) is the author/funder, who has granted bioRxiv a license to display the preprint in perpetuity. It is made available under aCC-BY-ND 4.0 International license.

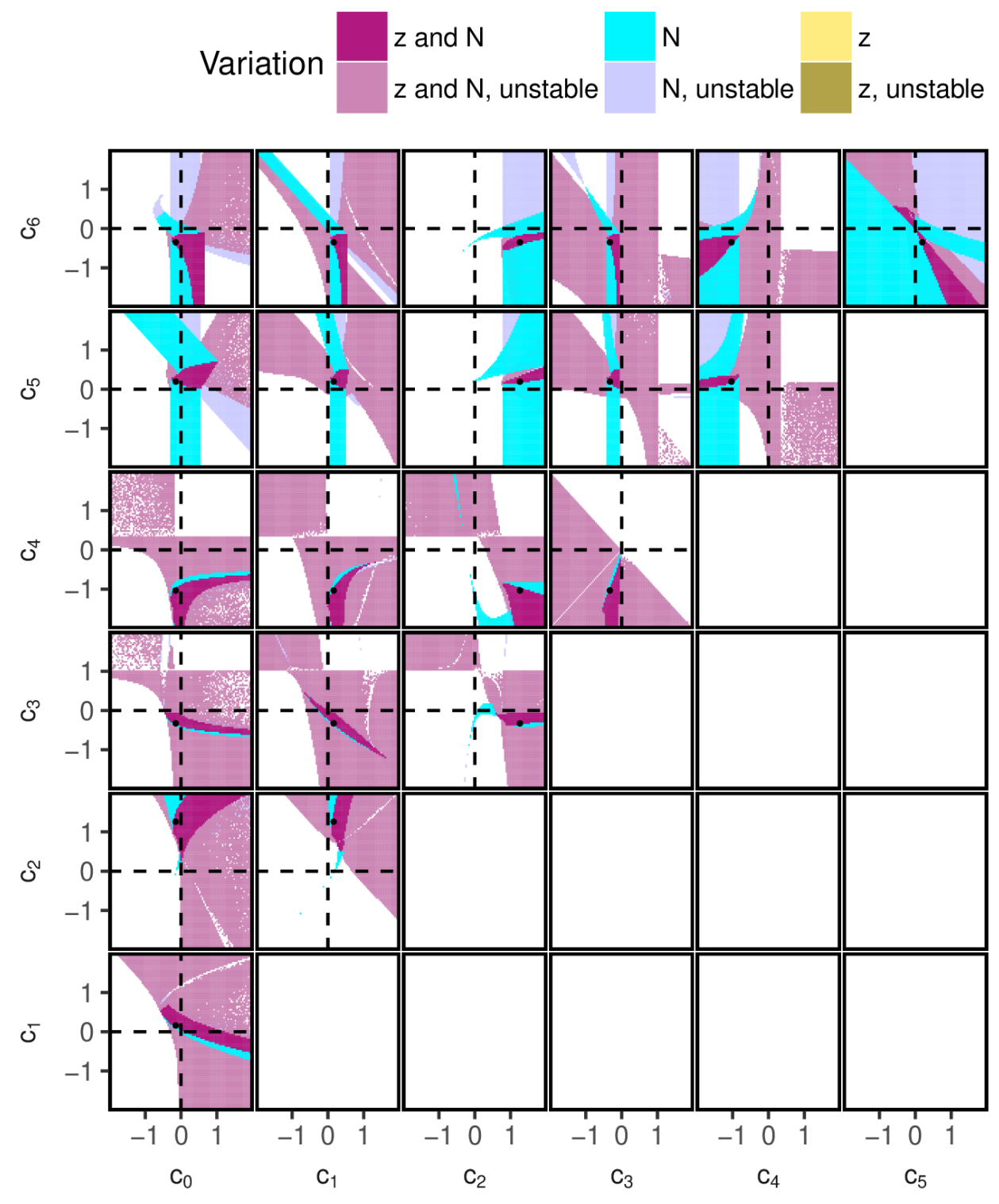

Figure S2.1: Regions in parameter space where spatial variation abundance and diversification of trait values can occur, including unstable equilibria. The shown regions are based on the properties of the asymmetric equilibria obtained by intersecting the ellipses with trait value $z=0$ and $z=1$ with the mirrored ellipses at both trait values (following equations 26) - (29). The values and stability of these ellipses were calculated using Mathematica. Only the upper diagonal graphs are shown. The white regions correspond to areas where no spatial variation in $N$ or $z$ was predicted in the system or where the predicted equilibrium contained negative values. The black dots correspond to the parameter settings that were used in the top panels of Fig. 7. The pixelated regions are due to the floating point precision in calculating the imaginary part. 


\section{S3. Additional example for the eco-evolutionary model}

836 Here, we show an additional example time series of the eco-evolutionary model. In this

${ }_{837}$ example, we have weakened selection by dividing both $c_{5}$ and $c_{6}$ by 10 . In order to speed

838 up selection in this example, we have increased the mutation rate $\mu$ to 0.05 . All other

839 parameters are as in Fig. 7. In this example, at both $z=0$ as well as $z=1$, the model

${ }_{840}$ has two equilibria, of which one is above $N_{\text {crit }}$ and the other below. 
(a)

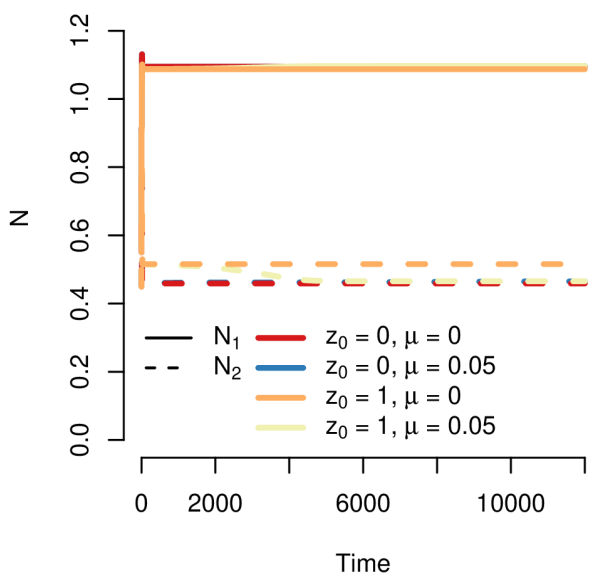

(c)

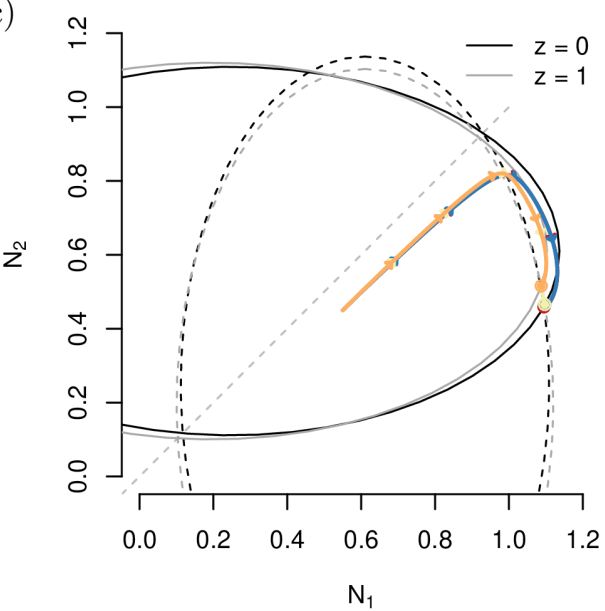

(b)

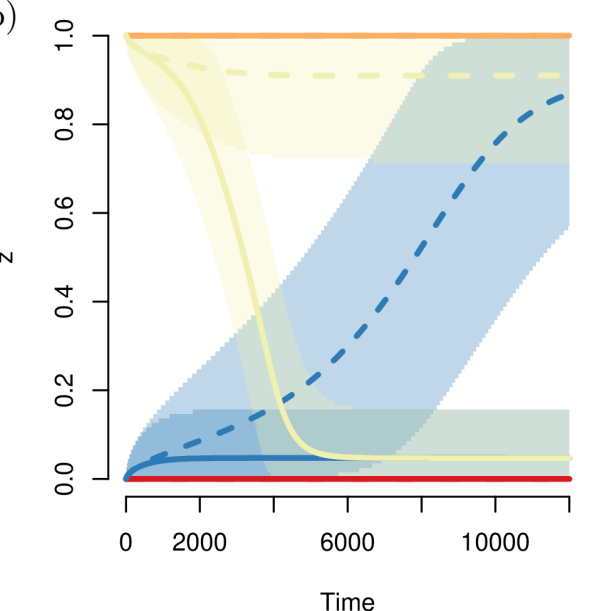

(d)

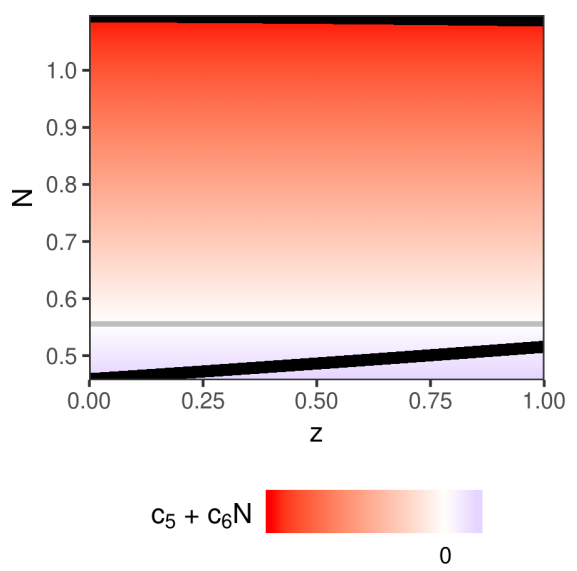

Figure S3.1: Example time series for the eco-evolutionary model. Different colors represent different model runs where mutations were either present $(\mu=0.05)$ or not $(\mu=0)$ and that started with a monomorphic population with either trait value $0\left(z_{0}=0\right)$ or $1\left(z_{0}=1\right)$. (a) Abundance time series, the red and blue trajectories largely overlap for the first 2000 time steps, as do the yellow and orange lines. (b) Corresponding mean trait values and the spread, shown as the regions in trait space that contained $95 \%$ of the individuals of each patch. For the scenarios without mutations (orange and red line), the trait values in both patches completely overlap. For the scenarios with mutations the trait values in the two patches diverge over time, regardless of the initial trait distribution. (c) Trajectories in phase space. Also drawn are the isoclines at $z=0$ (black) and $z=1$ (grey). (d) Equilibrium densities for monomorphic populations with trait value $z$. At any given value of $z$ two branches exist, indicating the two different densities that the two patches will tend to. In the background, the direction of selection at any given density is shown, with red values referring to selection for smaller trait values and blue colors to selection for larger trait values. The grey line corresponds to $N_{\text {crit }}$, the density at which selection vanishes. Parameter values: $c_{0}=-0.148, c_{1}=0.162, c_{2}=1.262, c_{3}=-0.326, c_{4}=-1.034, c_{5}=0.0194$ and $c_{6}=-0.03492$. 


\section{${ }_{841}$ S4. Migration between patches}

842 Differences in abundance between patches can be evened out through migration. If the

${ }_{843}$ same proportion of individuals in both patches migrates to the respective other patch,

844 the larger population will contribute more individuals to the smaller population and vice

845 versa. Hence, the difference in abundance between the patches is expected to decrease and

${ }_{846}$ the smaller patch should be less likely to go extinct. Furthermore, migration may hamper

$\mathbf{8 4 7}$ trait diversification through recurrent inflow of genes that have emerged through a se-

${ }_{848}$ lection pressure elsewhere. It is therefore vital to see whether the diversity in abundance

849 and trait values that emerged in our original model can be maintained under migration.

850 Ecological model

$\mathbf{8 5 1}$ First, we adapt the purely ecological model to include migration:

$$
\begin{aligned}
& \frac{d N_{1}}{d t}=f\left(N_{1}, N_{2}\right) \cdot N_{1}+m \cdot\left(N_{2}-N_{1}\right), \\
& \frac{d N_{2}}{d t}=f\left(N_{2}, N_{1}\right) \cdot N_{2}+m \cdot\left(N_{1}-N_{2}\right),
\end{aligned}
$$

${ }_{852}$ with $m$ being the fraction of individuals in each patch that migrate to the other patch.

${ }_{853}$ The fitness function $f$ is the same as the one used in the main text (equation 2). 
(a)

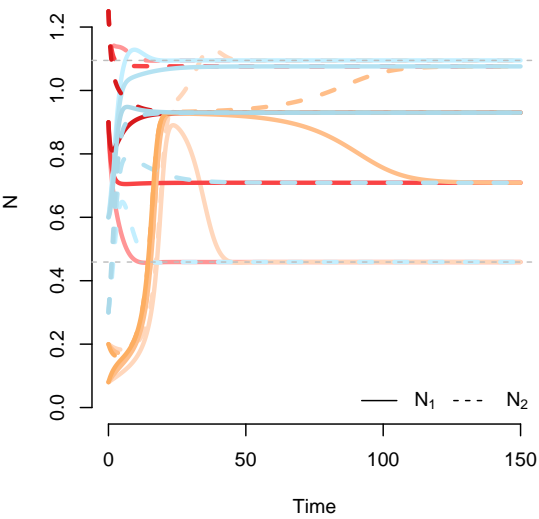

(c)

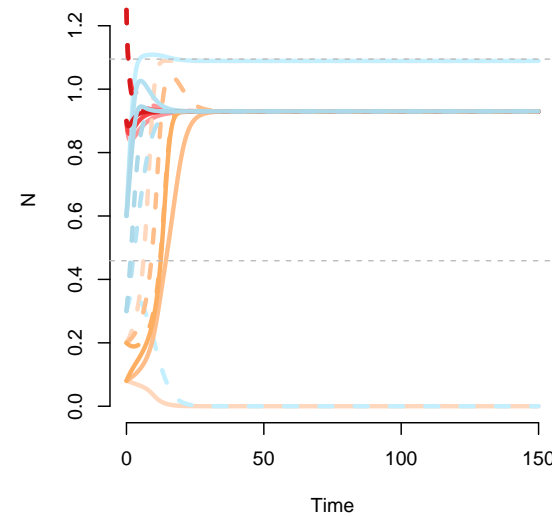

(b)

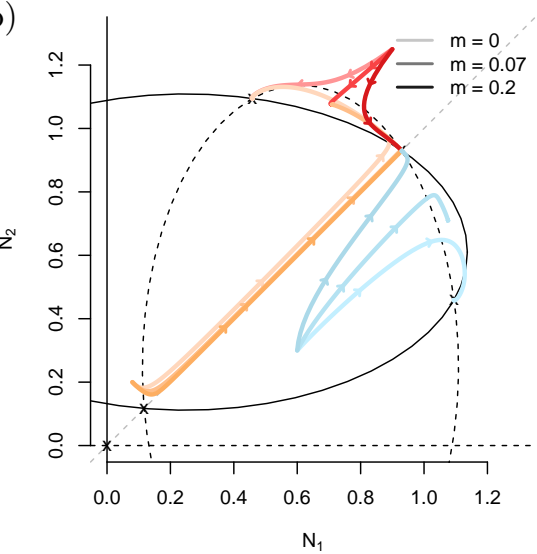

(d)

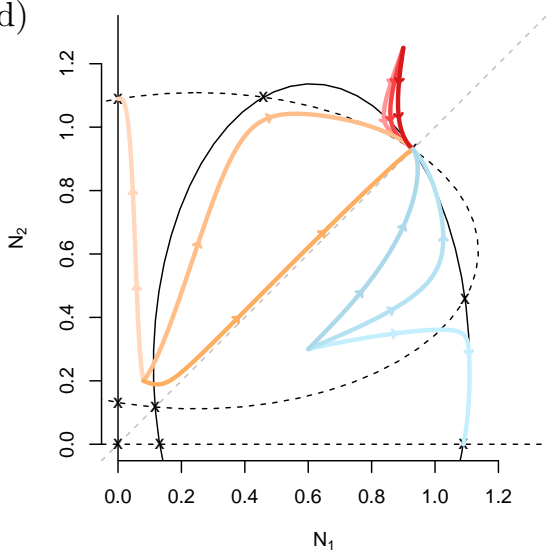

Figure S4.1: Time series of abundance for the ecological model with different migration rates. Otherwise, the parameter settings are as in the main text (see Fig. 4). The different colors correspond to different initial conditions, with the saturation indicating the migration rate. The equilibria and ellipses are shown for the case without migration.

We solved the model with migration numerically and compared the results to those without migration (Fig. S4.1). Migration increases the time it takes for the system to reach the asymmetric equilibrium (top left panel). Furthermore, migration affects the existence/stability and precise value of the equilibria (compare the red trajectories for

${ }_{858} m=0$ and $m=0.07$ in the top panels). This becomes apparent when plotting the

859 final abundances in both patches as a function of the migration rate (Fig. S4.2). At low

s60 migration rates, the two separate equilibria remain relevant. However, when the migration rate reaches a critical value (close to 0.10 for the depicted parameter combination), the 

$w_{1, b}=\left\{\begin{array}{ll}\frac{d n_{1, b}}{d t}=f\left(z_{b}, N_{1}, N_{2}\right) n_{1, b}-\mu n_{1, b}+\frac{\mu}{2} n_{1, b-1}+\frac{\mu}{2} n_{1, b+1}+m \cdot\left(n_{2, b}-n_{1, b}\right) & \text { if } 1<b<100 \\ \frac{d n_{1,1}}{d t}=f\left(z_{1}, N_{1}, N_{2}\right) n_{1,1}-\frac{\mu}{2} n_{1,1}+\frac{\mu}{2} n_{1,2}+m \cdot\left(n_{2,1}-n_{1,1}\right) & \text { if } b=1 \\ \frac{d n_{1,100}}{d t}=f\left(z_{100}, N_{1}, N_{2}\right) n_{1,100}-\frac{\mu}{2} n_{1,100}+\frac{\mu}{2} n_{1,99}+m \cdot\left(n_{2,100}-n_{1,100}\right) & \text { if } b=100\end{array}\right.$.

870 In our approach, we treat $m$ as a constant, such that migration is trait-independent.

871 From numerical solutions of the evolutionary model with migration (Fig. 9), it becomes

$\mathbf{8 7 2}$ apparent that the difference in average trait value is far more sensitive to migration than

873 the difference in abundance at the used parameter values: at $m=0.01$, the two patches 
${ }_{874}$ still reach different abundances, but they no longer obtain different average trait values.

875 Therefore, we show the final abundances of time series that were solved numerically

876 for up to $10^{4}$ time steps in two different graphs with different scales (Fig. S4.3). The

${ }_{877}$ differences in trait value disappear rapidly with increasing migration rates. This seems

878 to be caused by the relatively large influx of individuals from the larger patch that are

879 adapted to the density in their native patch, combined with a relatively low selection gradient. Interestingly, the effect of the migration rate depends on the mutation rate. This reflects that an increase in mutation rate affects the mutation-selection balance, causing the average trait value to shift away from the boundary values.

(a)

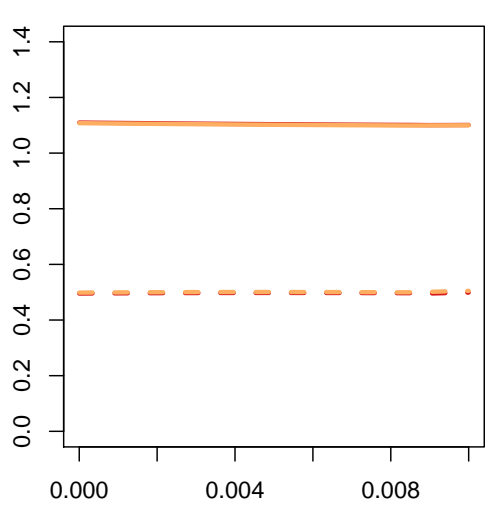

(c)

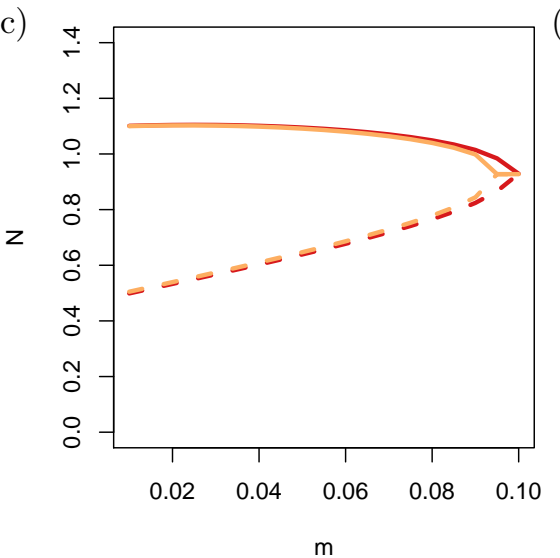

(b)

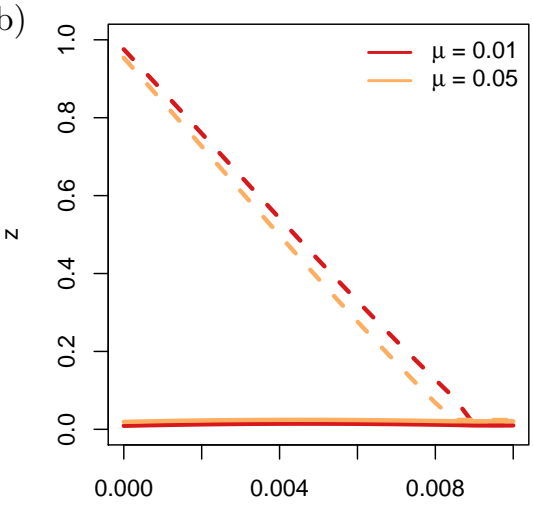

m

(d)

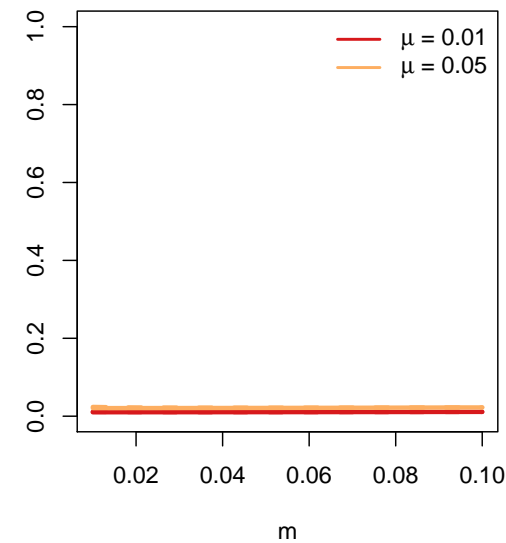

Figure S4.3: Final abundances in numerical solutions at time $t=10^{5}$, which was enough time for the system to reach equilibrium. The top and bottom half differ only in the range of migration rates that they contain. Mutation rate was varied, but all other parameter values were the same as in the evolutionary model in the main text. 

available under aCC-BY-ND 4.0 International license.

${ }_{883}$ In constructing Figure S4.3, we have re-assigned patch identity afterwards based on the

${ }_{884}$ final density (the patch with the larger final density was assigned to patch 1). Otherwise,

885 the lines would move back and forth between the two branches. That does mean however,

886 that the branches no longer correspond to a specific patch. 


\section{S5. Individual-based simulations}

${ }_{888}$ We tested the robustness of the results against demographic stochasticity with an individualbased model. This also allowed us to include diploid multi-locus genetics.

\section{Genetics} Genetics were diploid and consisted of a 10-locus system with 10 alleles per locus. Alleles were inherited independently across loci (no linkage). Every locus, contributed a value no factors other than genetics that affected the trait value.

897 Finally, for every newborn, there was a probability $\mu$ that it obtained a mutation at one of its loci. If a mutation was determined to occur, the locus and the value of the target allele were drawn from a uniform distribution, independently of the original allele value.

Fitness function

901

902

We assumed non-overlapping generations and the fitness function only affected female reproduction. This is one of the deviations from our original model, where males and females were not distinguished. For a female in patch 1, with trait value $z$, the number of offspring was drawn from a Poisson distribution with mean:

$$
\lambda=2 e^{c_{0}+c_{1} D_{1}+C_{2} D_{2}+c_{3} D_{1}^{2}+c_{4} D_{2}^{2}+c_{5} z+c_{6} z D_{1}},
$$

905 with $D_{i}$ the density in patch $i$. If patch $i$ has area $A_{i}$,

$$
D_{i}=\frac{N_{i}}{A_{i}}
$$

The factor of two in equation (S5.1) serves to compensate for the fact that males do not generate offspring. Instead, for every female that obtained offspring, the father of that clutch was randomly sampled among all males in the corresponding patch. Offspring were also born in the same patch, although in the model runs with migration, they had 
910 a small chance to move to the other patch. Although this process actually corresponds

$\mathbf{9 1 1}$ to dispersal, we use the term migration throughout this supporting information, for

912 consistence with the main text.

913 Finally, we had to set an area for the patches. For all model runs, the area was equal in

${ }_{914}$ both patches $\left(A_{1}=A_{2}=A\right)$. In the original model, the scaling of $N_{i}$ was arbitrary, and

${ }_{915} N_{i}$ could also take non-integer values. In the individual-based model this is not possible.

916 Here, $N_{i}$ can only take integer values. Changing the size of the area allows us to alter

917 the relative effect of demographic stochasticity, and thereby also the amount of genetic

918 drift in the system. When the area is large, the same equilibrium density corresponds to

$\mathbf{9 1 9}$ a larger absolute number of individuals. Hence, demographic stochasticity is expected to

920 have only a limited effect under these circumstances.

921 Initial population

922 The number of individuals in each patch was drawn from a normal distribution with

923 mean $N_{0}$ and standard deviation $\sigma=10$. For each of the patches, the fraction of males in

924 the initial population was drawn from a uniform distribution between 0.25 and 0.75 . The

925 number of individuals and the number of males were then rounded to the closest integer

$\mathbf{9 2 6}$ and applying the absolute value to avoid negative values (which rarely occurred because

$\mathbf{9 2 7}$ of sufficiently large $N_{0}$ ). Next, the allele values at the loci were also randomly sampled

928 from a uniform distribution: at every locus alleles were assigned, with equal probability

929 for every possible allele.

930 Runs and results

931 Direct averaging of the replicates proved difficult, due to stochasticity making it im${ }_{932}$ possible to predict which of the two patches would become the high density patch. In933 stead, we summarize the results qualitatively and show a few time series to illustrate ${ }_{934}$ the point. Throughout the simulation runs, we varied three parameters: $m \in[0,0.005]$, ${ }_{935} \mu \in[0.1,0.05]$, and $A \in[100,1000,5000]$. We tested all combinations of these parameter 936 settings and ran 10 replicates for each combination, for a total $2 \cdot 10^{5}$ time steps. If one ${ }_{937}$ of the two patches went extinct, the simulation was stopped. 
(a)

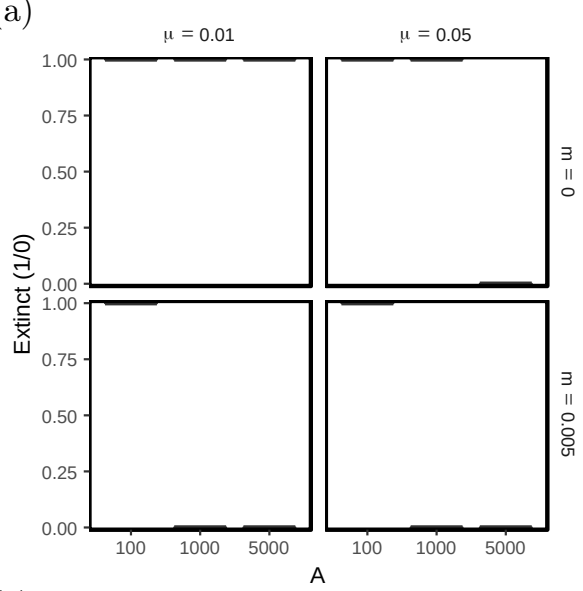

(c)

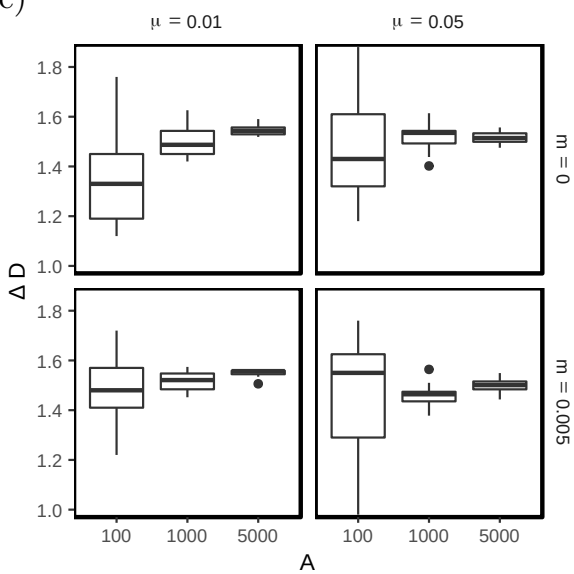

(b)

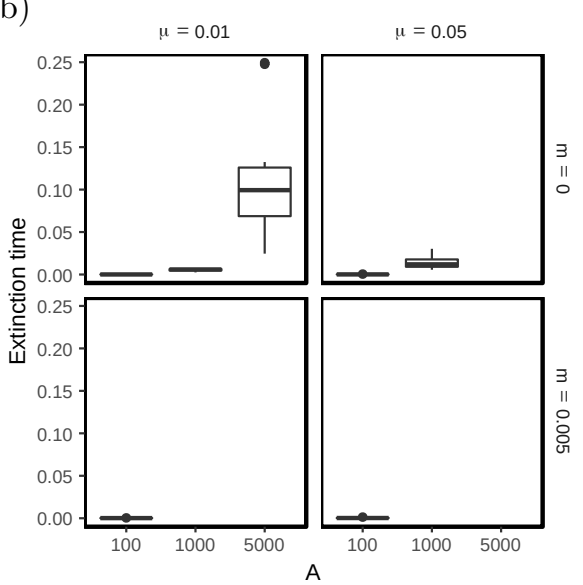

(d)

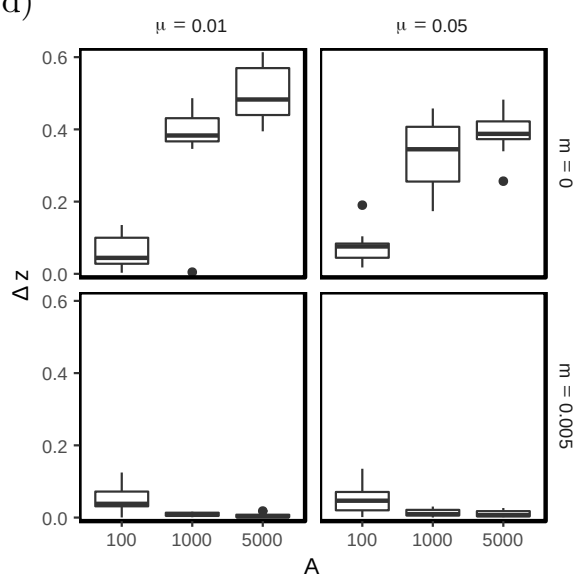

Figure S5.1: Summary of the runs from the individual-based model. Shown are boxplots that display (a) whether a patch in the replicates went extinct (1) or not (0), (b) the time at which this happened, (c) the difference in density between the patches at that point in time, and (d) the difference in average trait value between the patches at the end of the simulation $\left(2 \cdot 10^{5}\right.$ or extinction time). The parameter values of the fitness function were as in Fig. 7.

938 First of all, the results show a strong consistency across replicates, despite the presence 939 of demographic stochasticity. This stochasticity leads to extinction in all cases where 940 $A=100$ (Figure S5.1(a)). Furthermore, this panel also show that the mutation rate, and ${ }_{941}$ hence the potential speed of adaptation, has a direct effect on whether the populations can ${ }_{942}$ persist. Comparing panel (a) to (b), shows that at lower mutation rate, extinction occurs ${ }_{943}$ after approximately $10 \%$ of the simulation time, indicating that short-term co-existence of 
944 two patches with different abundances and trait value might be possible (as illustrated in

945 Figure S5.2). Panel (a) also shows that extinction is less likely when migration is allowed.

946 Migration has, however, only a very limited effect on the final difference in abundance

947 (panel $(d)$ ). In agreement with the results from SI S4, migration strongly affects the

${ }_{948}$ possibility of trait adaptation (panel (d), and Figure S5.3). The situation that is closest

949 to the deterministic evolutionary model that we presented in the main text consists of a

950 large patch size that reduces the effect of demographic stochasticity, combined with low

951 (no) migration and high mutation rates (for rapid adaptation). An example time series

952 of this case is shown in Figure S5.4.

(a)

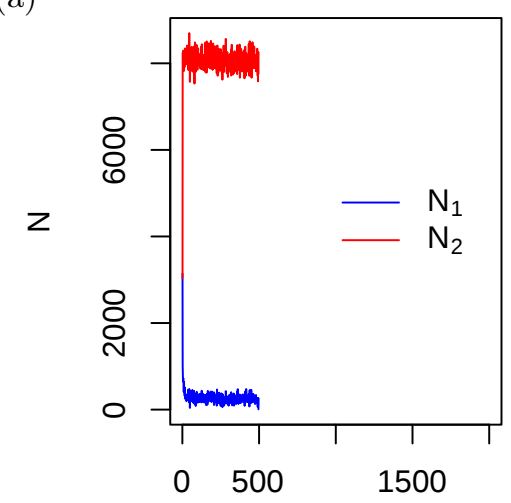

Time (b)

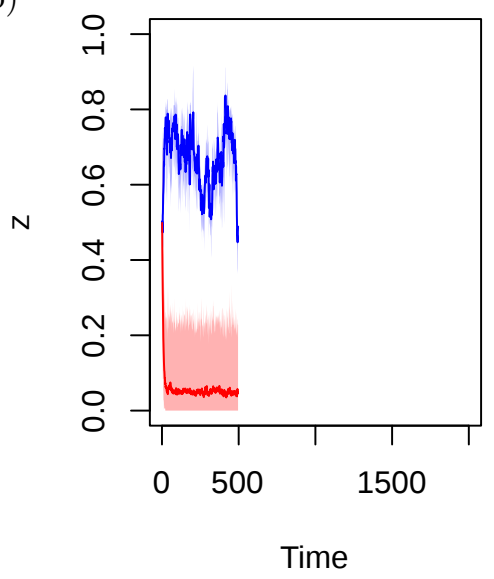

Figure S5.2: Time series run for one of the replicates with $\mu=0.01, m=0$ and $A=5000$. 
(a)

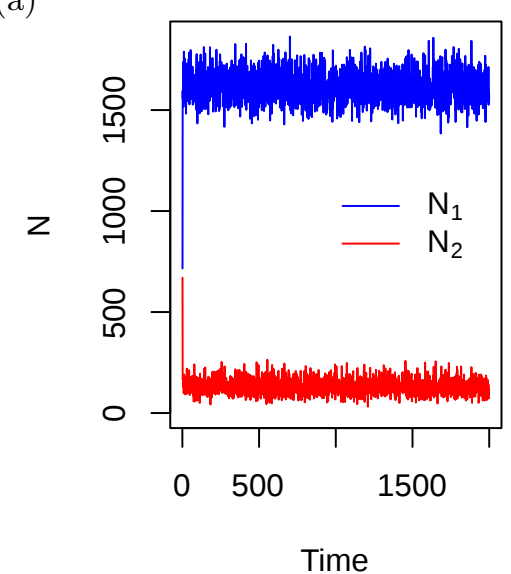

(b)

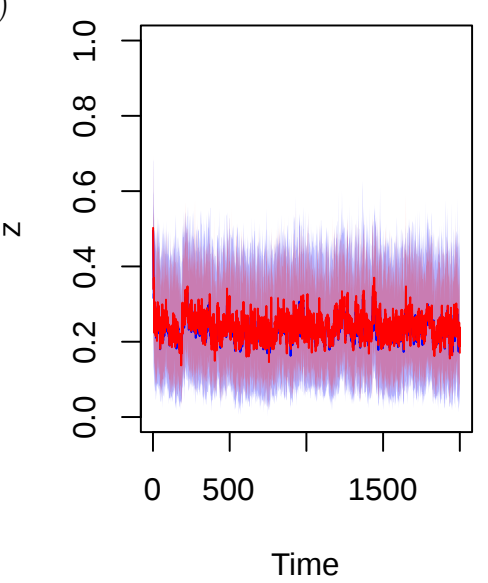

Figure S5.3: Time series run for one of the replicates with $\mu=0.05, m=0.005$ and $A=1000$.

(a)

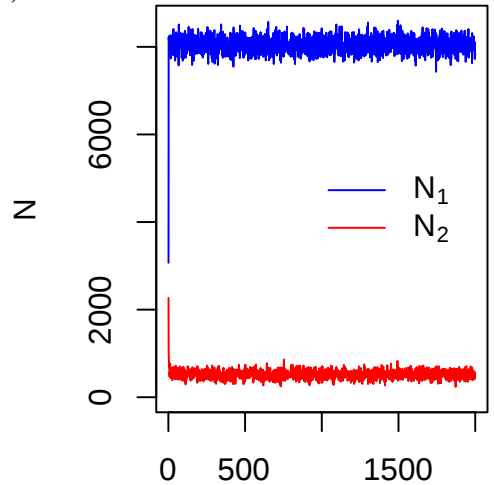

(b)

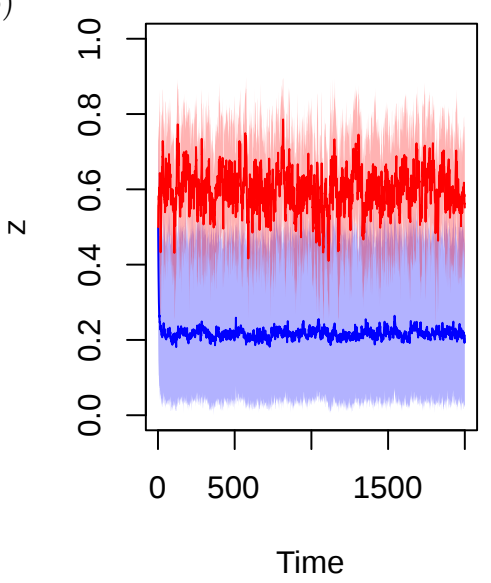

Figure S5.4: Time series run for one of the replicates with $\mu=0.05, m=0$, and $A=5000$. 